\title{
ANÁLISE FLORÍSTICA E ESTRUTURAL DA VEGETAÇÃO LENHOSA DO RIO COMEMORAÇÃO, PIMENTA BUENO, RONDÔNIA, BRASIL ${ }^{1}$
}

\author{
Izildinha S. MIRANDA
}

RESUMO - Estudou-se a composição floristica e estrutura da vegetação da região do Rio Comemoração, em Pimenta Bueno, Rondônia. O levantamento estrutural foi realizado em dez transectos distribuidos em floresta de terra firme (3), mata ciliar (5) e mata alagada (2). Utilizou-se o método Ponto Quadrante, com 50 pontos distantes $10 \mathrm{~m}$ entre si em cada transecto. Em cada ponto foram inventariadas quatro plantas com o limite minimo de $10 \mathrm{~cm}$ de CAP. Todas as comunidades estudadas mostraram distribuição de circunferência exponencial negativa. A riqueza de espécies e diversidade foram maiores nas florestas de terra firme; as matas ciliares apresentam em média as maiores áreas basais; as matas alagadas apresentaram as maiores estimativas de individuos/ha, embora as estimativas de área basal/ha fossem bastante baixas. Nas florestas de terra firme as principais espécies foram: Qualea paraensis, Maquira guianensis, Macrolobium acaciefolium e Dialium guianensis. Nas matas ciliares as principais espécies foram: Maquira guianensis, Macrolobium acaciefolium, Zygia latifolia, Couratari tenuicarpa, Mauritia flexuosa, Protium apiculatum, Parkia pamurensis, Oenocarpus bataua e Qualea paraensis. Nas matas alagadas as principais espécies foram: Pseudobombax cf. faroense, Qualea paraensis, Virola surinamensis, Clusia cf. planchoniana, Macrolobium angustifolium e Ferdinandusa guianensis. A grande maioria (92\%) das espécies foram encontradas em apenas um tipo fisionômico, apenas 30 espécies estavam em dois ou mais ambientes. A análise de similaridade e agrupamento revelam uma grande heterogeneidade florística e estrutural existente dentro e entre os três tipos fisionômicos.

Palavras-chave: Abundância, Florística, Fitossociologia, Vegetação lenhosa, Amazônia.

\section{Floristic and Structural Analysis of Wood Vegetation of Comemoração River, Pimenta} Bueno, Rondônia, Brazil

\begin{abstract}
The floristic composition and structure of the vegetation in the valley of the River Comemoração was studied.. The structural survey was carried out on ten transects distributed in terra firme forest (3), riparian forest (5) and open swamp forest (2). The Point centeredquarter method was used, with 50 sampling points, distributed $10 \mathrm{~m}$ from each other. At each point four plants were sampled, with the minimum limit of $10 \mathrm{~cm}$ circumference at breast height $(\mathrm{CBH})$. All the studied communities showed circumference distributions following a negative exponential curve. Species richness and diversity indices were largest in the terra firme forests; the riparian forests showed largest basal areas; and the swamp forests presented the largest number of individuals/ha, although the basal area/ha was low. In the terra firme forests the most important species were: Qualea paraensis, Maquira guianensis, Macrolobium acaciefolium and Dialium guianensis. In the riparian forests the most important species were: Maquira guianensis, Macrolobium acaciefolium, Zygia latifolia, Couratari tenuicarpa, Mauritia flexuosa, Protium apiculatum, Parkia panurensis, Oenocarpus bataua and Qualea paraensis. In the swamp forests the most important species were: Pseudobombax cf. faroense, Qualea paraensis, Virola surinamensis, Clusia cf. planchoniana, Macrolobium angustifolium and Ferdinandusa guianensis. The great majority $(92 \%)$ of the species were found in only one community, only 30 species were found in two or more communities. The similarity and cluster analysis revealed a great floristic and structural heterogeneity both within and among the three communities types.
\end{abstract}

Key-words: Abundance, Floristic composition, Phytosociology, Woody Vegetation, Amazon.

'Trabalho executado dentro do EIA-RIMA da UHE Rondon II, a ser construída pela Eletrogoes

S.A. EIA-RIMA conduzido pela Apidiá Planejamento Agropecuário Ltda, Porto Velho, RO. ${ }^{2}$ Faculdade de Ciências Agrárias do Pará, DCF, Av. Tancredo Neves, S/N, C.P. 917, CEP 66.077530, Belém, Pará. izildinha@usa.net 


\section{INTRODUÇÃO}

A vegetação que margeia o Rio Comemoração enquadra-se, numa escala de 1:5.000.000, nas florestas de transição encontradas nas áreas de tensão ecológica (IBGE, 1993), ou seja, no encontro de duas regiões ecológicas, a Floresta Ombrófila e a Savana (Cerrado). Muito pouco se conhece sobre as florestas de transição, principalmente em áreas de contato entre as duas principais formações vegetais brasileiras (Floresta Ombrófila Amazônica/ Cerrados do Planalto Central).

Segundo Veloso et al. (1991) a fisionomia das florestas de transição depende da escala estudada, pois em escalas menores é sempre possivel separar os diferentes tipos fisionômicos. No campo, pode-se verificar que a vegetação que margeia o Rio Comemoração apresenta cinco fisionomias distintas: duas fisionomias florestais predominantes, floresta de terra firme e mata ciliar; mata aberta alagada (ou mata alagada); pequenas manchas de savanas; e, algumas capoeiras (vegetação secundária).

$\mathrm{Na}$ área do Rio Comemoração será construida a Usina hidroelétrica Rondon II, que abastecerá a região do Município de Pimenta Bueno. Desta forma, este trabalho tem a finalidade de gerar subsidios científicos e contribuir para estratégias de preservação naquela área e em áreas circunvizinhas, objetivando a elaboração de uma lista de espécies encontradas na vegetação que margeia o Rio, a descrição das fisionomias encontradas e a caracterização da estrutura das florestas, utilizando indices como abundância, frequência, área basal e diversidade.

\section{METODOLOGIA}

\section{Área de Estudo}

O Rio Comemoração, localizado no municipio de Pimenta Bueno, Estado de Rondônia, situa-se no limite da Depressão Interplanáltica da Amazônia Meridional com o Planalto dos Parecis. Tem suas nascentes no Planalto dos Parecis e corre para o Rio Machado, afluente importante do Rio Madeira. A região caracteriza-se por apresentar uma superfície rebaixada, entalhada por drenagem incipiente que proporciona uma dissecação do relevo em colinas e interflúvios tabulares (RADAMBRASIL, 1978).

Os solos da região são classificados como Cambissolo Tropical Eutrófico associados a Areias Quartzosas (RADAMBRASIL, 1978). O clima é equatorial quente e úmido, com pluviosidade anual média entre 2.000 e $2.250 \mathrm{~mm}$, com longa estação chuvosa, mas significante estação seca. A temperatura anual média é em torno de $24^{\circ} \mathrm{C}$, apresentando, no inverno, o fenômeno das friagens com grande quedas de temperatura. Os meses de seca, entre maio e setembro, podem apresentar deficit hídrico, principalmente nos meses de julho a agosto (Nimer, 1991).

\section{Métodos de Campo}

Foram realizados dois tipos de inventários: (1) florístico, para compor 
uma lista de espécies ocorrentes nas diferentes fisionomias; (2) estrutural, para obtenção de parâmetros quantitativos que auxiliam na caracterização das fisionomias amostradas. Os inventários estruturais foram realizados apenas nas três fisionomias mais dominantes: a floresta de terra firme, a mata ciliar e a mata alagada.

Para análise da estrutura da vegetação utilizou-se o método Ponto Quadrante (Cottam \& Curtis, 1956). Foram demarcados 10 transectos: três em floresta de terra firme, cinco em mata ciliar e dois em mata alagada. Em cada transecto foram amostrados 50 pontos, estando os pontos distantes $10 \mathrm{~m}$ entre si. Em cada ponto foram inventariadas quatro plantas com circunferência à altura do peito (CAP) maior ou igual a $10 \mathrm{~cm}$, totalizando 200 individuos por transecto. Os transectos foram paralelos ao Rio, no sentido montante, e alternados entre as margens esquerda e direita, distantes entre si de 2,5 a 3,0 km (Tab. 1). De cada planta foram anotados a circunferência do caule e a distância da planta ao ponto.

Todas as espécies inventariadas foram coletadas e identificadas; aquelas férteis foram herborizadas e depositadas no Herbário do Museu Paraense Emilio Goeldi (MPEG). Foram realizadas coletas aleatórias de plantas férteis, dentro e fora dos transectos, incluindo-se ervas, arbustos, trepadeiras, epifitas e hemiparasitas.

\section{Métodos de Análises}

Para o estudo da estrutura da vegetação

seguiram-se as recomendações de Brower et al. (1998), considerando os seguintes indices: abundância $\left(\mathrm{n}_{\mathrm{i}}\right)$, densidade total (DT), densidade relativa (Dr), frequência relativa $(\mathrm{Fr})$, dominância relativa (Dor) e Índice de Valor de Importância (IVI). A estimativa da densidade total e consequentemente da dominância absoluta seguiu as recomendações de Pollard (1971) que apresenta estimativas menos tendenciosas das populações estudadas.

A diversidade de espécies foi calculada com o indice de Shannon $\left(\mathrm{H}^{\prime}\right)$. O indice de Shannon é amplamente utilizado por sua simplicidade e principalmente por possuir duas propriedades intrínsecas: (1) $H^{\prime}=0$ se e somente se a amostra tiver apenas uma espécie; (2) H' alcança o valor máximo quando todas as espécies forem igualmente abundantes (Magurran, 1988). O valor de H' é usualmente encontrado entre 1,5 e 3,5 e raramente ultrapassa 4,5. A equitabilidade (E) foi calculada segundo Pielou (1977). A equitabilidade expressa a relação entre a diversidade e o máximo valor que esta pode alcançar. A equitabilidade é obtida entre 0 e 1,0 , onde 1,0 representa a situação em que todas as espécies são igualmente abundantes. Tanto a diversidade de Shannon quanto a equitabilidade assumem que todas as espécies foram incluídas na amostragem (Magurran, 1988).

A similaridade floristica foi calculada utilizando-se o coeficiente de Jaccard (J) através de uma matriz 
Tabela 1. Localização dos transectos inventariados ao longo do Rio Comemoração, Município de Pimenta Bueno, Rondônia.

\begin{tabular}{|c|c|c|c|c|c|c|}
\hline Transecto & Fisionomia & Margem & Distância da margem & Latitude & Longitude & Attitude $(m)$ \\
\hline $\mathrm{T} 1$ & F. Terra firme & Direita & $500 \mathrm{~m}$ & $\mathrm{~S} 12^{\circ} 07^{\prime} 20,1^{\prime \prime}$ & W60³5'23,8" & 320 \\
\hline $\mathrm{T} 2$ & Mata Ciliar & Esquerda & $10 \mathrm{~m}$ & $S 1209^{\prime} 08,5^{\prime \prime}$ & W60\%36'21,7" & 200 \\
\hline $\mathrm{T} 3$ & Mata alagada & Esquerda & $1000 \mathrm{~m}$ & $S 12^{\circ} 06^{\prime} 09,1^{\prime \prime}$ & W60'36'44,2" & 200 \\
\hline T4 & Mata Ciliar & Direita & $10 \mathrm{~m}$ & $\mathrm{~S}_{12}{ }^{\circ 0} 4^{\prime 25}, 7^{\prime \prime}$ & W60 $96^{\circ} 49,9^{\prime \prime}$ & 200 \\
\hline T5 & FTerra firme & Direita & $300 \mathrm{~m}$ & $S 12^{\circ} 05^{\prime} 53,5^{\prime \prime}$ & W60 $3623,1^{\prime \prime}$ & 325 \\
\hline T6 & Mata Ciliar & Esquerda & $10 \mathrm{~m}$ & $\mathrm{~S} 12^{202} 47,0^{\prime \prime}$ & W60\%3739,0" & 225 \\
\hline$\pi$ & Mata Cillar & Direita & $10 \mathrm{~m}$ & $S 1200^{\prime} 46,5^{\prime \prime}$ & W60 $40^{\circ} 18,2^{\prime \prime}$ & 210 \\
\hline T8 & F.Terra firme & Direita & $300 \mathrm{~m}$ & $S 1201^{\prime} 57,0^{\prime \prime}$ & W60'38'44,7" & 300 \\
\hline T9 & Mata alagada & Esquerda & $600 \mathrm{~m}$ & $S 12^{\circ 01} 37,0^{\prime \prime}$ & W60 $60^{\circ} 00,4^{\prime \prime}$ & 200 \\
\hline T10 & Mata Ciliar & Direita & $10 \mathrm{~m}$ & $S 1200000,4^{\prime \prime}$ & W60 $41^{\prime} 42,0^{\prime \prime}$ & 236 \\
\hline
\end{tabular}

binária de presença e ausência. O indice de Jaccard pode variar de 0 a 1 e a similaridade aumenta com o aumento do índice; em geral, Jaccard acima de 0,5 indica alta similaridade (Ludwig \& Reynolds, 1988).

A análise de agrupamento foi realizado pelo método hierárquico, aglomerativo e politético, utilizando-se a distância euclideana como medida de dissimilaridade (Milligan \& Cooper, 1987). O dendrograma foi produzido aplicando o método de Wards. Segundo Ludwig \& Reynolds (1988), a análise de agrupamento baseada na estrutura de abundância das espécies forma grupos que podem delimitar diferentes comunidades bióticas.

\section{RESULTADOS E DISCUSSÃO Composição Florística e Descrição Fisionômica}

Existe uma grande riqueza de espécies ao longo do Rio Comemoração. Um total de 383 espécies, distribuídas em 84 famílias, foram identificadas em 535 amostras coletadas (Tab. 2). As famílias predominantes em número de espécies foram: Melastomataceae (22 espécies), Caesalpiniaceae (20), Rubiaceae (15), Fabaceae (14), Guttiferae (13), Mimosaceae (13), Annonaceae (12), Lauraceae (12), Moraceae (11), Sapotaceae (11), Chrysobalanaceae

Malpighiaceae (10) e Myrtaceae (10). Os gêneros predominantes em número de espécies foram: Inga ( 9 espécies), Miconia (8), Licania (8), Aspidosperma (5), Guarea (5), Micropholis (5) e Mouriri (5).

A grande maioria $(65 \%)$ das 
Tabela 2. Lista de espécies do Rio Comemoração, Município de Pimenta Bueno, Rondônia. Habito: $\mathrm{A}=$ árvore; $\mathrm{AB}=$ arbusto; $\mathrm{E}=$ erva; $\mathrm{T}=$ trepadeiras e lianas; $\mathrm{EP}=$ epifita; $\mathrm{HP}=$ hemiparasita. Habitat: $\mathrm{TF}=$ floresta de terra firme; $\mathrm{MC}=$ mata ciliar; $\mathrm{MA}=$ mata alagada; $\mathrm{S}=$ savana; $\mathrm{C}=$ capoeira.

\begin{tabular}{|c|c|c|c|c|}
\hline Familias & Espécie & Nome Vulgar & Habito & Habitat \\
\hline Acanthaceae & Justicia angustifolia Lindl. & & $E$ & MC \\
\hline Acanthaceae & Justicia sp. & & $\mathrm{E}$ & $T-F$ \\
\hline Acanthaceae & Justicia sp. & & $\mathrm{E}$ & MC \\
\hline Anacardiaceae & Anacardium giganteum Hanc. Ex Engl & Cajuí & A & MC \\
\hline Anacardiaceae & Anacardium tenuifolium Ducke & Cajuaçu & A & T.F \\
\hline Anacardiaceae & Astronium lecointei Ducke & Jitó preto & A & MC \\
\hline Anacardiaceae & Tapirira guianensis Aubl. & & A & $T-F$ \\
\hline Annonaceae & Anaxagorea acuminata (Dun.) St. Hill. Ex A.DC. & Envira preta da casca grossa & A & MC \\
\hline Annonaceae & Annona paraensis R.E.Fries & Envira & A & MC \\
\hline Annonaceae & Duguettia calycina Benoist & Envira pitinga & A & MC \\
\hline Annonaceae & Guatteria chrysopetala (Steud.) M.q. & & A & MC \\
\hline Annonaceae & Guatteria duckeana R.E.Fries & envira caju & A & $T-F$ \\
\hline Annonaceae & Guatteria foliosa Benth & Envira branca & A & $T \cdot F$ \\
\hline Annonaceae & Guatteria microsperma R. \& Fr. & & A & MC \\
\hline Annonaceae & Guatteria poeppigiana Mart. & Envira da casca verde & A & MC \\
\hline Annonaceae & Oxandra xylopioides Diels & Envira vassourinha do cerrado & A & MA \\
\hline Annonaceae & Xylopia benthamii R.E.Fries & Envira araticum & A & MC \\
\hline Annonaceae & Xylopia ct. calophyila R. \& Fries & Envira vassourinha & A & $\mathrm{T}-\mathrm{F}$ \\
\hline Annonaceae & Xylopia nitida Dunal & Envira amargosa & A & MC \\
\hline Apocynaceae & Aspidosperma cf. eteinbachii M.G.F. & & A & MC \\
\hline Apocynaceae & Aspidosperma ct. verruculosum Muell Arg. & & A & T-F \\
\hline Apocynaceae & Aspidosperma cylindrocarpon Muell Arg. & Peroba do cerrado & A & T-F \\
\hline Apocynaceae & Aspidosperma desmanthum Benth ex Muell Arg. & & A & MC \\
\hline Apocynaceae & Aspidosperma tomentosum C. Martius & & A & T.F \\
\hline Apocynaceae & Galactophora catycina (Huber) Woodson. & & $\mathrm{T}$ & $\mathrm{s}$ \\
\hline Apocynaceae & Himatanthus articulatus (Vahi) Woodson & Sucuba & A & $\mathrm{s}$ \\
\hline Apocynaceae & Malouetia ct. lata Mgf. & Sorvinha & A & T-F \\
\hline Apocynaceae & Mandevilla hirsuta (R. \& Sch.) K.Schum. & & $\mathrm{T}$ & MC \\
\hline Aquifoliaceae & Mex inundata Poepp. & casca esverdeada & A & MA \\
\hline Aquifoliaceae & Ilex parvifiora Benth & Anil & A & MC \\
\hline Araceae & Anthurium bonplandii & & E & MA \\
\hline Araceae & Anthurium kunthii Poeppig & & EP & MC \\
\hline Araceae & Heteropsis spruceana Schott. & & $T$ & MC \\
\hline Araceae & Monstera andansonii Schott. & & EP & MC \\
\hline Araceae & Philodendron distantilobum Krause & & EP & T-F \\
\hline Araceae & Philodendron solimoesense A.C. Smith & & EP & MC \\
\hline Araliaceae & Gilibertia cuneata March & & $A B$ & MA \\
\hline Araliaceae & Gilibertia palustris Ducke & Urucurana & A & T-F \\
\hline Araliaceae & indeterminada & & $E$ & T-F \\
\hline Araliaceae & Indeterminada & & A & T.F \\
\hline Araliaceae & Indeterminada & & E & MC \\
\hline Araliaceae & Indeterminada & & $A B$ & $T-F$ \\
\hline Araliaceae & Schefflera morototoni (Aublet) Frodin & & $A B$ & $\mathrm{~s}$ \\
\hline
\end{tabular}


Tabela 2 - Continuação

\begin{tabular}{|c|c|c|c|c|}
\hline Caesalpinaceae & Tachigalia paniculata Aubl. & Tachi preto da mata alagada & A & TF/MC \\
\hline Caryocaraceae & Anthodiscus amazonicus & Piquiá & A & MC \\
\hline Cecropiaceae & Cecropia concolor Willd. & Embauba de anel & A & MC \\
\hline Cecropiaceae & Coussapoa angustifolia Aubl. & Três pernas & A & MC \\
\hline Cecropiaceae & Pouroma bicolor Mart. & Embaubarana & A & T.F \\
\hline Cecropiaceae & Pouroma velutina Maquel & Embauba & A & $T-F$ \\
\hline Celastraceae & Goupia glabra Aubl. & Cupiuba/Cupluba da folha grande & A & MC \\
\hline Celastraceae & Maytenus guianensis K.L. & Coral & A & $T \cdot F$ \\
\hline Chloranthaceae & Hedyosmum brasiliense & & A & MA \\
\hline Chrysobalanaceae & Hirtella bicornis Mart. \& Zucc & Macucuzinho & A & T-F \\
\hline Chrysobalanaceae & Hirtella excelsa Standl. & & A & $T \cdot F$ \\
\hline Chrysobalanaceae & Hirtella racemosa Lam. & Macucu da folha pequena & A & MC \\
\hline Chrysobalanaceae & Licania apetala (E.Mey) Fritsch. & Papo de nambu & A & MC \\
\hline Chrysobalanaceae & Licania canescens R.Ben. & & A & MC \\
\hline Chrysobalanaceae & Licania gardneri (Hook.F.) Fritscb. & Macucu casca fina & A & MA \\
\hline Chrysobalanaceae & Licania heteromorpha Benth & Macucu vermelho/ vermelhăo & A & MC \\
\hline Chrysobalanaceae & Licania incana Aubl. & Macucu da folha pequena & A & T.F \\
\hline Chrysobalanaceae & Licania membranacea Sagot. ex Laness & Macucu casca dura & A & TF/MC \\
\hline Chrysobalanaceae & Licania octandra (Hoffmgg. ex R.T.S.) Kuntze & & A & MC \\
\hline Chrysobalanaceae & Licania sclerophylla (Mart. ex Hook) Frits & & A & $\mathrm{s}$ \\
\hline Commelinaceae & Floscopa peruviana Hassk & & $E$ & MC \\
\hline Connaraceae & Connarus punctatus Planchon & & $\mathrm{AB} / \mathrm{T}$ & MC \\
\hline Connaraceae & Connarus ruber (P. \& E.) Planc. & & T & MC \\
\hline Convolvulaceae & Bonamia peruviana V.O.Ostr. & & $\mathrm{T}$ & $\mathrm{S}$ \\
\hline Convolvulaceae & Dicranostyles sp. & & T & MC \\
\hline Convolvulaceae & ipomoea setifera Polr & & $T$ & MC \\
\hline Convolvulaceae & Maripa scandens Aubl. & & $T$ & MC \\
\hline Dichapetalaceae & Tapura guianensis Aubl. & Mata mata amarela & A & MC \\
\hline Dilleniaceae & Davilla kunthii St. Hil. & & $T$ & $\mathrm{~S}$ \\
\hline Dilleniaceae & Davilla pedicellaris Benth & & $\mathrm{T}$ & $\mathrm{s}$ \\
\hline Dilleniaceae & Doliocarpus dentatus (Aubl.) Standi. & & $\mathrm{AB} / \mathrm{T}$ & MC \\
\hline Dioscoreaceae & Dioscorea huberi L.I. & & $\mathrm{T}$ & $\mathrm{S}$ \\
\hline Ebenaceae & Diospyros duckei Sand. & Envira preta casca grossa & A & T-F \\
\hline Elaeocarpaceae & Sloanea garckeana K.Schum & Orelha de gato & $\mathrm{A} / \mathrm{AB}$ & MC \\
\hline Elaeocarpaceae & Sloanea grandiflora Smith & Casca dura preta da mata alagada & A & MA \\
\hline Elaeocarpaceae & Sloanea cf. parviflora Planch. ex Benth & Orelha de onça folha grande & A & T.F \\
\hline Elaeocarpaceae & Sloanea tuerckeimii Donn SM. & Orelha de onça & A & MC \\
\hline Ericaceae & Gaylussacia amazonica Huber & & $A B / E$ & $\mathrm{~S}$ \\
\hline Eriocaulaceae & Paepalanthus bifidus (Schard) Kunth & & $\mathrm{E}$ & MC \\
\hline Eriocaulaceae & Syngonanthus bisumbellatus (Steud.) Ruhl. & & $E$ & S \\
\hline Eriocaulaceae & Syngonanthus oblongus (K.)Ruhl. & & $E$ & MA \\
\hline Erythroxylaceae & Erythroxylum anguifugum Mart. & Ingá xixica & A & T.F \\
\hline Erythroxylaceae & Erythroxylum mucronatum Benth & & A & $T \cdot F$ \\
\hline Euphorbiaceae & Amanoa guianensis Aubl. & Casca de açai/ Araçaí & A & MC \\
\hline Euphorbiaceae & Aparisthmium cordatum (Juss.) Baill. & & A & T.F \\
\hline Euphorbiaceae & Caryodendron amazonicum Ducke & & A & MA \\
\hline Euphorbiaceae & Croton sp. & Maravovô & A & MC \\
\hline Euphorbiaceae & Hevea brasiliensis (Willd. ex Adr.) M.Arg. \& L. & Seringa & A & $T \cdot F$ \\
\hline
\end{tabular}


Tabela 2 - Continuação

\begin{tabular}{|c|c|c|c|c|}
\hline Euphorbiaceae & Mabea angustifolia Spruce ex Bentham & Taquari & $A B$ & c \\
\hline Euphorbiaceae & Mabea fistulifera Mart. & Taquari & A & $\mathrm{MC} / \mathrm{C}$ \\
\hline Euphorbiaceae & Pera distichophylla (Mart.) Baillon & Jatobarana & A & MC \\
\hline Euphorbiaceae & Sandwithia guianensis & & $A B$ & T.F \\
\hline Fabaceae & Canavalia grandiflora Benth & & $\mathrm{T}$ & MC \\
\hline Fabaceae & Centrosema bifidum Benth & & $\mathrm{T}$ & s \\
\hline Fabaceae & Dalbertia gracilis Benth & & $T$ & MC \\
\hline Fabaceae & Derris floribunda (Benth) Ducke & & $T$ & C \\
\hline Fabaceae & Derris sp. & Timbó preto & $\mathrm{T}$ & MC \\
\hline Fabaceae & Dioclea of. violacea Mart. ex Benth & & $T$ & MC \\
\hline Fabaceae & Diplotropis brasiliensis (Tul.) Benth & Sucupira amarela & A & T.F \\
\hline Fabaceae & Dipteryx odorata (Aubl.) Willd. & Cumaru ferro & A & T-F \\
\hline Fabaceae & Hymenolobium nitidum Benth & Angelim da folha grande & A & MC \\
\hline Fabaceae & Hymenolobium sp. & Angelim pedra & A & MC \\
\hline Fabaceae & Machaerium madeirense Pitt & & $T$ & T-F \\
\hline Fabaceae & Machaerium of. quinata & & $T$ & MC \\
\hline Fabaceae & Plerocarpus rohrii Vahl & Mututi branco & A & MC \\
\hline Fabaceae & Vataireopsis speciosa Ducke & Angelim amargoso & A & MC \\
\hline Flacourtiaceae & Casearia javitensis H.B.K. & & A & MC \\
\hline Flacourtiaceae & Casearia pitumba Sleumer & & A & MC \\
\hline Flacourtiaceae & Casearia sylvestris SW. & Breu de tucano & A & MC \\
\hline Gentianaceae & Irlbachia alata (Aubl.) Maas & & $E$ & $\mathrm{~s}$ \\
\hline Gramineae & Ichnanthus axilaris (Nees) Hitch. \& Chase & & $E$ & MC \\
\hline Gramineae & Olyra longifolia H.B.K. & Taboquinha & $A B$ & MC \\
\hline Gramineae & Orthoclada laxa (L.Rich.)Beauv. & & $\mathrm{E}$ & $T-F$ \\
\hline Guttiferae & Calophyllum brasiliense Camb. & Macacarecuia & A & T.F \\
\hline Guttiferae & Caraipa densifolia Mart. & & $A B$ & $s$ \\
\hline Guttiferae & Clusia cf. planchoniana Engl. & Apul amarelo & A & MA \\
\hline Guttiferae & Clusia pana-panari (Aubl.)Choisy & parece aroeira & A & MA \\
\hline Guttiferae & Kielmeyera rosea (Spring) Mart. & & $A B$ & $\mathrm{~S}$ \\
\hline Guttiferae & Rheedia brasiliensis (Mart.) PI. et Tr. & Bacuri de anta & A & MC \\
\hline Guttiferae & Rheedia macrophylla (Mart.) Pl. et Tr. & Bacuri & A & $\mathrm{MC}$ \\
\hline Guttiferae & Symphonia globulifera L.f. & Bacunzinho & A & MA/MC \\
\hline Guttiferae & Tovomita brevistaminea Engl. & Pau de fogo & A & T.F \\
\hline Guttiferae & Tovomita cf. speciosa Ducke & & A & MC \\
\hline Guttiferae & Tovomita cf. triflora Huber & Barba de lontra 2 & A & T-F \\
\hline Guttiferae & Vismia cayannensis (Jacq.) Pers & Lacre branco & A & MC \\
\hline Guttiferae & Vismia macrophylla H.B.K. & Lacre branco & A & $\mathrm{MC} / \mathrm{S}$ \\
\hline Heliconiaceae & Heliconia julianii Barreiros & Bico de tucano & $E$ & MC \\
\hline Heliconiaceae & Heliconia psittacorum L.F. & & $E$ & MC \\
\hline Hernandiaceae & Sparathanthelium tupiniquinorum Mart. & Cedro mara & A & MC \\
\hline Hippocrateaceae & Cheiloclinium cognata (Miers) A.C. Sw. & Gogó amarelo & A & MC \\
\hline Hippocrateaceae & Hippocralea ovata Lam. & & $T$ & MC \\
\hline Hippocrateaceae & Salacia cognata (Miers) Peyr & & $T$ & MC \\
\hline Hippocrateaceae & Salacia impressifolia (Miers) A.C. Smith & & $T$ & TF/MC \\
\hline Hippocrateaceae & Salcia sp. & Macucu casca grossa & A & TF/MC \\
\hline Humiriaceae & Humiria balsamifera (Aubl.) St.Hil. & & A & $\mathrm{S}$ \\
\hline Lacistemaceae & Lacistema aggregatum (Berg) Rusby & & A & MC \\
\hline
\end{tabular}


Tabela 2 - Continuação

\begin{tabular}{|c|c|c|c|c|}
\hline Lauraceae & Aniba parvifiora (Meiss) Mez & Louro branco da folha grande & A & MC \\
\hline Lauraceae & Licaria armeniaca (Nees) Kost & Louro branco/Pipo de macaco & A & TF/MC \\
\hline Lauraceae & Mezilaurus aff. subcordata (Ducke) Kosterm & Itaubinha & A & $T \cdot F$ \\
\hline Lauraceae & Mezilaurus synandra (Mez) Kosterm & Itauba amarela & A & $T \cdot F$ \\
\hline Lauraceae & Mezilaurus itauba (Meissn) Mez & Itaubarana & A & MC \\
\hline Lauraceae & Nectandra amazonum Nees & Louro branco & A & MC \\
\hline Lauraceae & Nectandra cuspidata Nees & & $A B$ & C \\
\hline Lauraceae & Nectandra lucida Nees & Louro amarelo & A & MA \\
\hline Lauraceae & Ocotea canaliculata Mez & Louro amarelo & A & T-F \\
\hline Lauraceae & Ocotea gracilis (Meissn) Mez & & $A B$ & T.F \\
\hline Lauraceae & Ocotea guianensis Aubl. & Louro folha fina - Louro imbé & A & T.F \\
\hline Lecythidaceae & Cariniana micrantha Ducke & Cachimbeiro & A & MC \\
\hline Lecythidaceae & Couratari tenuicarpa AC. Smith & Tauari mirim & A & MC \\
\hline Lecythidaceae & Eschweilera coriacea (A.P. DC.) Mart. ex Berg. & Mata mata casca grossa & A & T-F \\
\hline Linaceae & Hebepetalum humirifolium (Planch) Benth & parece cachuá & A & MATTF \\
\hline Linaceae & Roucheria calophylla Planch & & A & MC \\
\hline Loganiaceae & Potalia amara Aubl. & & $A B$ & MC \\
\hline Loganiaceae & Strychnos amazonica Krukoff & Moreira & A & MC \\
\hline Loganiaceae & Strychnos peckii B.L.Robinson & & T & MC \\
\hline Loranthaceae & Phoradendron botryostachys Eichl. & Erva de passarinho & HP & T-F \\
\hline Loranthaceae & Phoradendron crassifolium (DC) Eichl. & Erva de passarinho & $\mathrm{HP}$ & $\mathrm{MC}$ \\
\hline Loranthaceae & Psittacanthus of, collum-cyani Eichl. & Erva de passarinho & HP & MC \\
\hline Lythraceae & Cuphea melvilla Lindley & & $\mathrm{E}$ & $M C$ \\
\hline Malpighiaceae & Banisteriopsis schwannioides (Gr.)Gates & & $\mathrm{T}$ & MC \\
\hline Malpighiaceae & Banisteriopsis sp. & & $T$ & $\mathrm{~s}$ \\
\hline Malpighiaceae & Byrsonima ct. melanocarpa Ducke & & AB & $\mathrm{S}$ \\
\hline Malpighiaceae & Byrsonima stipulacea Adr. Juss & Murici & A & MC \\
\hline Malpighiaceae & Byrsonima umbellata Adr. Juss. & & $A B$ & S \\
\hline Malpighiaceae & Mascagnia anisopetala (Adr.Juss.) Griseb. & & $T$ & C \\
\hline Malpighiaceae & Mascagnia cornifolia (Adr. Juss,) Griseb. & & $\mathrm{T}$ & $\mathrm{s}$ \\
\hline Malpighiaceae & Stigmatophyllum palmatum (Cav.) Adr.Juss. & & $\mathrm{T}$ & MC \\
\hline Malpighiaceae & Tetrapteryx styloptera A. Juss. & & $\mathrm{T}$ & s \\
\hline Malpighiaceae & Tetrapteryx sp. & & $\mathrm{T}$ & MC \\
\hline Maranthaceae & Calathea zingiberina Koern & & $\mathrm{E}$ & MC \\
\hline Maranthaceae & Monotagnia laxum (Poepp. \& Endl.) K. Schum & Arroz cauaçu & $E$ & T-F \\
\hline Melastomataceae & Acisanthera $s p$. & & $E$ & S \\
\hline Melastomataceae & Bellucia grossularioides (L.) Tr. & Golaba de anta & A & MC \\
\hline Melastomataceae & Graffenrieda floribunda $\mathrm{Tr}$. & Goiaba de anta do cerrado & A & MA \\
\hline Melastomataceae & Henriettea ovata Cogn. & & A & MC \\
\hline Melastomataceae & Macairea pachyphylla Benth & Murta cascuda & A & MAVS \\
\hline Melastomataceae & Macairea theresiae Cogn. & & $E$ & S \\
\hline Melastomataceae & Miconia burchelli Triana & Murta branca & A & $T-F$ \\
\hline Melastomataceae & Miconia chrysophylla (Rich.) Urb. & Murta vermelha & A & TF/MC \\
\hline Melastomataceae & Miconia dispar Benth & & A & MC \\
\hline Melastomataceae & Miconia melinonis Navd. & & A & MC \\
\hline Melastomataceae & Miconia parviflora (Aubl.) Karst & & $A B$ & MC \\
\hline Melastomataceae & Miconia splendens (SW.) Griseb. & & A & MC \\
\hline
\end{tabular}


Tabela 2 - Continuação

\begin{tabular}{|c|c|}
\hline Melastomataceae & Miconia tetraspermoides Wurdack \\
\hline Melastomataceae & Miconia tomentosa (Rich) Don ex Dc. \\
\hline Melastomataceae & Mouriri angulicosta Morley \\
\hline Melastomataceae & Mouriri apiranga Spruce ex Triana \\
\hline Melastomataceae & Mouriri cf. callocarpa Ducke \\
\hline Melastomataceae & Mouriri duckeana Morley \\
\hline Melastomataceae & Mouriri myrtifolia Spruce ex Triana \\
\hline Melastomataceae & Tibouchina aspera Aublet \\
\hline Melastomataceae & Tibouchina stenocarpa (DC) Cogn \\
\hline Melastomataceae & Tococa guianensis Aubl. \\
\hline Meliaceae & Guarea carinata Ducke \\
\hline Meliaceae & Guarea cf. grandiflora DC. \\
\hline Meliaceae & Guarea cf. guidonia Sleum \\
\hline Meliaceae & Guarea subsessilifiora C.DC. \\
\hline Meliaceae & Guarea velutina A.Juss. \\
\hline Meliaceae & Trichilia lecointei Ducke \\
\hline Meliaceae & Trichilia micrantha Benth \\
\hline Meliaceae & Trichilia schomburgkii C.DC. \\
\hline Menispermaceae & Anomospermum reticulatum Mart. \\
\hline Mimosaceae & Adenophora (Ducke) Barneby \& Grimes \\
\hline Mimosaceae & Inga cayennensis Benth \\
\hline Mimosaceae & Inga ct. cinnanomea Spruce \\
\hline Mimosaceae & Inga disticha Benth \\
\hline Mimosaceae & Inga falcistipula Ducke \\
\hline Mimosaceae & Inga ingoides (Rich) Willd. \\
\hline Mimosaceae & Inga marginata Willd. \\
\hline Mimosaceae & Inga nitida Willd. \\
\hline Mimosaceae & Inga rubiginosa (Rich) Dc. \\
\hline Mimosaceae & Inga velutina (Poiret) Willdenon \\
\hline Mimosaceae & Parkia panurensis Spruce ex H.C. Hopkins \\
\hline Mimosaceae & Zygia caulifiora (Willid.) Killip. \\
\hline Mimosaceae & Zygia latifolia \\
\hline Monimiaceae & Siparuna guianensis Aubl. \\
\hline Moraceae & Brosimum acutifolium Huber \\
\hline Moraceae & Brosimum guianense (Aubl.) Huber \\
\hline Moraceae & Brosimum potabile Ducke \\
\hline Moraceae & Ficus catappaefolia Kunth et Barch \\
\hline Moraceae & Ficus trigona L.F. \\
\hline Moraceae & Maquira calophylla (P. \& E.) C.C.Berg. \\
\hline Moraceae & Maquira guianensis Aubl. \\
\hline Moraceae & Perebea mollis (Poeppig \& Endl.) Huber \\
\hline Moraceae & Pseudomedia laevigata Trec. \\
\hline Moraceae & Pseudomedia murure Standley \\
\hline Moraceae & Sorocea guilleminiana Gaud. \\
\hline Myristicaceae & Campsoneura ulei Warb. \\
\hline Myristicaceae & Iryanthera jurvensis Warb. \\
\hline Myristicaceae & Iryanthera sagotiana (Benth) Warb \\
\hline Myristicaceae & Virola calophylla Warb \\
\hline
\end{tabular}

\begin{tabular}{|c|c|c|}
\hline & $A B$ & MC \\
\hline \multirow[t]{2}{*}{ Murta da folha grande } & A & MC \\
\hline & A & MC \\
\hline Araça branco & A & MC \\
\hline \multirow[t]{2}{*}{ Araça casca fina } & A & T.F \\
\hline & A & MC \\
\hline \multirow[t]{3}{*}{ Araça da folha pequena } & A & MC \\
\hline & $\mathrm{AB}$ & $\mathrm{s}$ \\
\hline & $A B$ & $\mathrm{~S}$ \\
\hline \multirow[t]{8}{*}{ Murta branca da folha grande } & A & TF/MC \\
\hline & A & T-F \\
\hline & A & MC \\
\hline & A & T.F \\
\hline & $\mathrm{AB}$ & MA \\
\hline & A & T.F \\
\hline & $A B$ & C \\
\hline & $A B$ & $T-F$ \\
\hline \multirow[t]{2}{*}{ Andirobarana } & A & T-F \\
\hline & $T$ & MC \\
\hline Faveira fofa & A & T.F \\
\hline Ingarana vermelha & A & MC \\
\hline Ingá branco & A & MC \\
\hline Ingarana vermelha & A & MC \\
\hline Ingá vermelha & A & $T-F$ \\
\hline Ingá da beira do rio/ ingá cípó & A & MC \\
\hline Ingá amarela & A & MA \\
\hline Ingá branca & A & MC \\
\hline Ingá & A & MC \\
\hline Ingá peluda & A & MC \\
\hline Faveira fola & A & TF/MC \\
\hline Ingarana/ Ingarana amarela & A & MC \\
\hline Ingarana & A & MC \\
\hline Cacatinguia & A & $\mathrm{MC} / \mathrm{C}$ \\
\hline Mururé da folha grande & A & MC \\
\hline Muiratinga da folha pequena & A & MC \\
\hline Garrote & A & T.F \\
\hline Apui amarelo & A & MC \\
\hline Apui da folha pequena & A & MC \\
\hline Muiratinga & A & $T-F$ \\
\hline Mururé branco & A & MC \\
\hline Cauchorana & A & MC \\
\hline Pama da folha pequena & A & T-F \\
\hline Pama da folha grande & A & T-F \\
\hline Pama fura-fura & A & MC \\
\hline Caferana amarela & A & TF/MC \\
\hline Mirassacaca & A & T.F \\
\hline Mirassacaca & A & $T \cdot F$ \\
\hline Virola branca folha grande & A & $T \cdot F$ \\
\hline
\end{tabular}


Tabela 2 - Continuação

\begin{tabular}{|c|c|}
\hline Myristicaceae & Virola theiodora (Spr. ex Bth) Warb. \\
\hline Myrsinaceae & Cybianthus amplus (Mez in Engl.) Agostini \\
\hline Myrsinaceae & Rapanea guianensis Aubl. \\
\hline Myrtaceae & Calyptranthus creba McVaugh \\
\hline Myrtaceae & Eugenia feijoi Berg. \\
\hline Myrtaceae & Eugenia patrisii Vahl. \\
\hline Myrtaceae & Eugenia prosoneura Berg. \\
\hline Myrtaceae & Marlierea cf. velutina Mac Vangh \\
\hline Myrtaceae & Myrcia cf. deflexa (Poiret) DC. \\
\hline Myrtaceae & Myrcia fallax (Rich) DC. \\
\hline Myrtaceae & Myrcia sphaerocarpa DC. \\
\hline Myrtaceae & Myrciaria dubia (H.B.K.) McVaugh \\
\hline Myrtaceae & Myrciaria floribunda (Willd.) Berg. \\
\hline Nyctaginaceae & Neea ovalilolia Spruce ex J.A. Schimidt \\
\hline Nyctaginaceae & Neea sp. \\
\hline Nyctaginaceae & Neea sp. \\
\hline Ochnaceae & Ouratea discophora Ducke \\
\hline Ochnaceae & Ouratea paraensis Huber \\
\hline Ochnaceae & Ouratea polygyna Engl. \\
\hline Ochnaceae & Sauvagesia fruticosa Mart. et Zucc. \\
\hline Olacaceae & Chanouchiton kapleri \\
\hline Olacaceae & Dulacia candida (Poeppig.) O.Kuntze \\
\hline Olacaceae & Dulacia guianensis (Engl.) O.Kuntze \\
\hline Olacaceae & Heisteria barbata Cuat. \\
\hline Olacaceae & Heisteria laxiflora Engl. \\
\hline Olacaceae & Minquartia guianensis Aubl. \\
\hline Onagraceae & Ludwigia leptocarpa (Nutt) Hara \\
\hline Orchidaceae & Lockhartia goyazensis Rochb. f. \\
\hline Orchidaceae & Epistephium parviflorum Lindl. \\
\hline Passifloraceae & Passiffora coccinea Aubl. \\
\hline Piperaceae & Piper colubrinum Link \\
\hline Polygalaceae & Bredemeyera altissima (Poepp et Endl) Benn \\
\hline Polygalaceae & Bredemeyera floribunda Willd. \\
\hline Proteaceae & Panopsis rubescens (Pohl) Pitter \\
\hline Quilinaceae & Lacunaria jenmani (Oliv.) Ducke \\
\hline Rapateaceae & Rapatea pycnocephala Seub. \\
\hline Rhizophoraceae & Sterigmapetalum obovatum Kuklm \\
\hline Rubiaceae & Faramea longifolia Bth. \\
\hline Rubiaceae & Chomelia malaneoides M.Arg. ex Chav. \\
\hline Rubiaceae & Diodia ct. teres Walt. \\
\hline Rubiaceae & Ferdinandusa guianiae Spruce \\
\hline Rubiaceae & Ferdinandusa paraensis Ducke \\
\hline Rubiaceae & Genipa americana L. \\
\hline Rubiaceae & Ixora pubescens Willd \\
\hline Rubiaceae & Malanea malaneoides M.Arg. ex Chav. \\
\hline Rubiaceae & Pagamea coriacea (Spruce) Benth \\
\hline Rubiaceae & Pagamea guianensis Aubl. \\
\hline Rubiaceae & Palicourea quadrifolia (Rudge) Steyerm. \\
\hline Rubiaceae & Palicourea roseiflora Schum ex Kr. \\
\hline Rubiaceae & Remigia amazonica Schum. \\
\hline
\end{tabular}

\begin{tabular}{|c|c|c|}
\hline \multirow[t]{3}{*}{ Virola vermelha } & A & $T-F$ \\
\hline & $A \cup A B$ & $\mathrm{MC} / \mathrm{MA}$ \\
\hline & A & MA \\
\hline \multirow[t]{8}{*}{ Goiabăo } & A & MC \\
\hline & $\mathrm{AB}$ & MC \\
\hline & A & MC \\
\hline & $A B$ & T.F \\
\hline & A & MC \\
\hline & A & MC \\
\hline & A & MC \\
\hline & $A B$ & S \\
\hline \multirow[t]{2}{*}{ folha miuda } & A & MA \\
\hline & A & MC \\
\hline Cinzeiro folha pequena & A & T.F \\
\hline Cinzeiro & A & T-F \\
\hline \multirow[t]{2}{*}{ Joāo mole } & A & MC \\
\hline & A & T.F \\
\hline \multirow[t]{3}{*}{ parece uchi liso } & $A / A B$ & MC \\
\hline & A & MC \\
\hline & $\mathrm{E}$ & $\mathrm{s}$ \\
\hline Sem cheiro & A & MA \\
\hline \multirow[t]{2}{*}{ Envira catinga } & A & MC \\
\hline & A & MA \\
\hline \multirow[t]{2}{*}{ Catuaba de indio } & A & T.F \\
\hline & A & MC \\
\hline \multirow[t]{4}{*}{ Acari } & A & MC \\
\hline & $E$ & MC \\
\hline & EP & MC \\
\hline & $E$ & MA \\
\hline \multirow[t]{4}{*}{ Maracujá do campo } & $T$ & C \\
\hline & EP & MC \\
\hline & $A B$ & $\mathrm{~s}$ \\
\hline & $T$ & MC \\
\hline \multirow[t]{3}{*}{ Pau renda } & A & $\mathrm{MC} / \mathrm{TF}$ \\
\hline & A & T-F \\
\hline & $E$ & MA \\
\hline Rajadão & A & MC \\
\hline Osso de porco & A & T-F \\
\hline \multirow[t]{2}{*}{ Cipó de espinho } & A & MC \\
\hline & $E$ & $\mathrm{~S}$ \\
\hline Botão avermelhado & $A B$ & MA \\
\hline Itaubarana & A & TF/MA \\
\hline \multirow[t]{3}{*}{ Genipapo } & A & MC \\
\hline & A & TF/MC \\
\hline & $T$ & MC \\
\hline \multirow[t]{5}{*}{ Apurui do cerrado } & A & MA \\
\hline & $A B$ & $\mathrm{~s}$ \\
\hline & $\mathrm{E}$ & MC \\
\hline & $A B$ & $\mathrm{~s}$ \\
\hline & $A B$ & S \\
\hline
\end{tabular}


Tabela 2 - Continuação

\begin{tabular}{|c|c|c|c|c|}
\hline Rubiaceae & Retiniphyilum truncatum Muell.Arg. & & $A B$ & MA \\
\hline Rubiaceae & Uncaria guianensis Aubl. & & $T$ & MC \\
\hline Rutaceae & Hortia sp. & Genitá branco & A & MC \\
\hline Rutaceae & Rhabdondendron amazonicum (Spr. ex Benth) Hub. & & A & T.F \\
\hline Rutaceae & Sohnreya excelsa Krause & & A & $T-F$ \\
\hline Rutaceae & Zanthoxylum of. annulatum & Marupá amarelo & A & $T-F$ \\
\hline Rutaceae & Zanthoxylum sprucei A.Engler & Marupá preto & A & $T \cdot F$ \\
\hline Sabiaceae & Meliosma herbertii Rolfe & & $A B$ & MC \\
\hline Sapindaceae & Cupania rubiginosa (Poir) Radlk & & A & MC \\
\hline Sapindaceae & Matayba arborescens (Aubl.) Radlk & Pau de fenda & ANAB & TF/C \\
\hline Sapindaceae & Matayba inelegans (Spruce) Radlk. & & A & MA \\
\hline Sapindaceae & Paullinia coloptera Radlk & & $\mathrm{T}$ & MC \\
\hline Sapindaceae & Serjania paucidentata DC. & & $T$ & C \\
\hline Sapindaceae & Talisia pedicellaris Radlk. & & A & MC \\
\hline Sapindaceae & Toulicia cf. patentinervis Radlk. & Parece Pitombeira & A & $\mathrm{MC} / \mathrm{TF}$ \\
\hline Sapotaceae & Elaeoluma glabrascens & & A & MA \\
\hline Sapotaceae & Micropholis acutangula (Ducke) Eyma & & A & $T \cdot F$ \\
\hline Sapotaceae & Micropholis cyrtobotria (Mart. et Miq) Baill. & Abiu vermelho & A & $\mathrm{MC} / \mathrm{TF}$ \\
\hline Sapotaceae & Micropholis guianensis Pierre & & A & TF/MC \\
\hline Sapotaceae & Micropholis melinoniana Pierre & Falso bacuri & A & T.F \\
\hline Sapotaceae & Micropholis venulosa (Mart. et Eich.) Pierre & Vermelhinho de leite & A & T.F \\
\hline Sapotaceae & Myrtiluma eugenilolia (Pierre) Baill & Abiurana da folha grande & A & MC \\
\hline Sapotaceae & Pouteria caimito (R. et P.) Radlk & Abiurana amarela & A & MC \\
\hline Sapotaceae & Pouteria gromerata Miq. & Abiu branco & A & TF/MC \\
\hline Sapotaceae & Pouteria reticulata (Engl.) Eyma & Maçarandubinha & A & T.F \\
\hline Sapotaceae & Prieurella prieurii (A. DC.) Aubr. & Abiurana vermelha/ abiurana amarela & A & TF/MC \\
\hline Simarubaceae & Simaruba amara Aublet & & $A B$ & S \\
\hline Sterculiaceae & Sterculia speciosa K.Schum & Chichá & A & MC \\
\hline Sterculiaceae & Theobroma speciosum Willid. & Cacauí & A & MC \\
\hline Styracaceae & Styrax guianensis A.DC. & & A & MC \\
\hline Tiliaceae & Luehea speciosa Willd. & Chicharana & A & MA \\
\hline Tiliaceae & Luehea sp. & Chicharana & A & MC \\
\hline Tiliaceae & Lueheopsis cf. rosea (Ducke) Burret & Chicharana & A & MA \\
\hline Violaceae & Leonia glycycarpa Ruiz et Pav. & Cachuá & A & MC \\
\hline Violaceae & Rinorea cf. fiana (DC) Kuntz & & $\mathrm{AB}$ & $T \cdot F$ \\
\hline Violaceae & Rinorea guianensis Aublet & Falso murure & $A B$ & MC \\
\hline Vitaceae & Cissus erosa Rich & & $T$ & s \\
\hline Vochysiaceae & Qualea acuminata Spruce ex Warming & Cupiubinha/ Cambará casca grossa & A & TF/MC \\
\hline Vochysiaceae & Qualea dinizii Ducke & & A & S \\
\hline Vochysiaceae & Qualea paraensis Ducke & Cupiuba branca/ Cambará casca fina & A & T.F \\
\hline Vochysiaceae & Qualea wittrockii Malme & Parece araça do brejo & A & MA \\
\hline Vochysiaceae & Vochysia obscura Warm & & A & MC \\
\hline Vochysiaceae & Vochysia surinamensis Statleu & & A & MC \\
\hline Vochysiaceae & Vochysia vismiaetolia Warm & Cedrinho/Vela branca & A & TF/MC \\
\hline Xyridacea $\theta$ & Xyris jupicai L.C.Rich. & & $E$ & $\mathrm{~s}$ \\
\hline Xyridaceae & Xyris malmeana L.B.Smith & & $E$ & MC \\
\hline Xyridaceae & Xyris mima Smith \& Downs & & $E$ & $\mathrm{~s}$ \\
\hline Zingiberaceae & Costus arabicus L. & & $E$ & MC \\
\hline
\end{tabular}


espécies foram arbóreas, devido às coletas do inventário quantitativo nas fisionomias florestais. As demais espécies apresentaram habito arbustivo (48 espécies), herbáceo (32), trepadeira (46), epífitas (6) e hemiparasitas (3) (Tab. 2).

As florestas de terra firme, sobre latosolos vermelho-amarelos, ocorrem nos interflúvios, principalmente na margem direita do Rio Comemoração, onde o relevo se torna suavemente ondulado. $\mathrm{O}$ dossel alcança cerca de 15 a $20 \mathrm{~m}$ e as árvores emergentes, principalmente Dialium guianensis, Qualea paraensis, Aspidosperma tomentosum e Qualea dinizii, alcançam entre 20 a $25 \mathrm{~m}$. O estrato herbáceo é dominado por Maranthaceae como Calathea zingiberina, Monotagnia laxum (arrozcauaçu) e regeneração de espécies arbóreas. São também elementos comuns do estrato herbáceo as palmeiras acaules, como Geonoma acaulis (ubim) e Geonoma sp. (Ticum), e Phenakospermum guianensis (banana brava ou sororoca).

As matas ciliares ocorrem nos terrenos aluviais, estacionalmente alagados. O dossel alcança cerca de 15 $\mathrm{m} \mathrm{e}$ as árvores emergentes, principalmente Maquira guianensis, Couratari tenuicarpa e Macrolobium acaciefolium, podem alcançar até 25 m. No dossel destacam-se as palmeiras como Mauritia flexuosa (buriti) e Iriartella setigera (paxiubinha), comumente encontradas na região mais a jusante do Rio;
Euterpe precatoria (açai) e Oenocarpus bataua (patauá), que predominam nas matas ciliares mais a montante da região estudada. O estrato herbáceo é composto principalmente por Heliconiaceae, como Helliconia julianii (bico de tucano), e regeneração de espécies arbóreas; em alguns locais onde o relevo é suavemente rebaixado, formando grandes poças temporárias, é comum encontrarmos Olyra longifolia (taboquinha) em grande abundância. A grande abundância de trepadeiras, lianas e epífitas heliófilas encontradas, principalmente na margem do Rio, tais como Dalbergia gracilis, Dioclea cf. violacea, Stigmatophyllum palmatum e Paulinia coloptera, contribuem para a fisionomia bem caracteristica das matas ciliares dos rios amazônicos.

As matas abertas alagadas (ou matas alagadas) sobre solo hidromórfico, ocorrem principalmente na margem esquerda, atrás das matas ciliares, mais a jusante do Rio. A média de altura é em torno de $6 \mathrm{~m}$, as copas são bem verticais, permitindo boa luminosidade até o estrato herbáceo, que é denso com muitas Gramíneas, Xyridáceas, Eriocauláceas e Pteridófitas, etc. A arvoreta Pseudobombax cf. faroense se destaca em altura, podendo alcançar até $10 \mathrm{~m}$.

As pequenas manchas de savanas arbustivas ocorrem sobre areias quartzosas em terrenos mais planos nos interflúvios. Fisionomicamente são espécies arbóreas, Himatanthus articulatus, 
Kielmeyera tomentosum, Eugenia feijoi e Sclerolobium aureum se destacam nos campos naturais, não pela sua abundância, mas por constituirem os poucos elementos arbóreos existentes nessa fisionomia. Entre as trepadeiras, Davilla pedicellaris, Dioscorea huberi e Centrosema bifidum predominam.

Algumas pequenas áreas de vegetação secundária (capoeira) ocorrem principalmente na margem esquerda do Rio, com espécies características de áreas antropizadas, tais como: Serjania paucidentata, Passiflora coccinea, Cissus erosa, Derris floribunda, Mascagnia anisopetala e Mascagnia cornitolia.

\section{Distribuição de Tamanho}

Em todos os transectos estudados as comunidades arbóreas mostraram distribuição de circunferência (CAP) exponencial negativa, onde um grande número de individuos pequenos predominam (Fig.1). Essa distribuição é característica de vegetações naturais (Hartshorn, 1978), onde predominam populações estáveis e autoregenerativas (Whitmore, 1975).

Os dois transectos inventariados na mata alagada apresentaram o maior número de individuos na primeira classe de CAP (de 10 a $19 \mathrm{~cm}$ ), com poucos ou nenhum individuos nas classes superiores (Fig. 1), confirmando a fisionomia mais arbustiva existente na área. As espécies Macrolobium angustifolium, Pseudobombax cf. faroense, Mauritia flexuosa, Qualea paraensis e Virola surinamensis apresentaram indivíduos com CAP acima de $60 \mathrm{~cm}$.

Nas florestas de terra firme, as espécies que apresentaram os maiores CAP foram: Tachigalia myrmecophylla, Parkia panurensis, Qualea paraensis, Qualea dinizii e Macrolobium acaciefolium. Nas matas ciliares as espécies com maiores CAP foram: Hevea brasiliensis, Maquira guianensis, Couratari tenuicarpa, Macrolobium acaciefolium e Coussapoa angustifolia.

\section{Abundância e Diversidade}

As riquezas de espécies e diversidades foram maiores nas florestas de terra firme; as matas ciliares apresentam em média menos individuos/ha e maiores áreas basais do que as florestas de terra firme. As mata alagadas apresentaram os menores números de espécies e menores diversidades e equitabilidade; no entanto, apresentaram as maiores estimativas de indivíduos/ha, com distância média entre as árvores bem pequena $(1,6$ e $1,4 \mathrm{~m}$, nos dois transectos) embora as estimativas de área basal/ha fossem bastante baixas (Tab. 3). Esses dados mostram a existência de muitos indivíduos arbustivos na mata alagada. Os índices de equitabilidade de todos os transectos foram maiores que 0,5 , indicando que a diversidade máxima é próxima da diversidade encontrada (Magurran, 1988).

As diversidades encontradas nas três fisionomias estudadas foram ligeiramente inferiores aos indices de diversidade encontrados em outras 


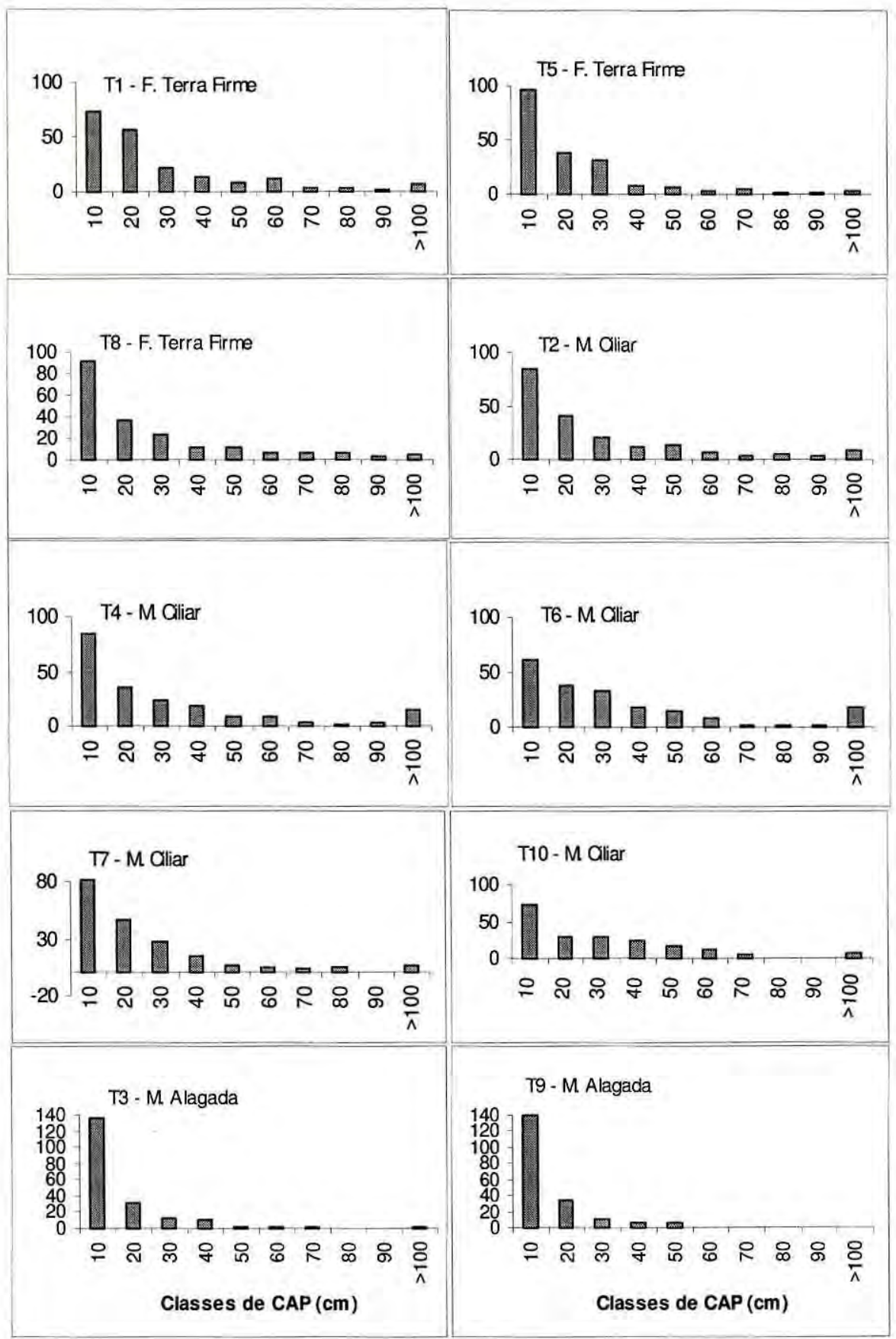

Figura 1. Distribuição de freqüência das classes de circunferência à altura do peito (CAP) das árvores amostradas nos dez transectos estudados em três fisionomias no Rio Comemoração, Rondônia. 
Tabela 3. Riqueza (S), Diversidade (H'), Equitabilidade (E), Estimativa da densidade total (DT - indivíduos/ha) e Estimativa da área basal total ( $\mathrm{AB}$ - área basal $/ \mathrm{ha})$ das árvores $(\mathrm{CAP} \geq 10 \mathrm{~cm})$ inventariadas em 10 transectos de ponto quadrante ( 50 pontos) amostrados no Rio Comemoração, Município de Pimenta Bueno, Rondônia.

\begin{tabular}{ccccccc}
\hline Transecto & Fisionomia & S & H & E & DT & AB(m2) \\
\hline T1 & F.Terra firme & 71 & 3.888 & 0.912 & 2140.29 & 38.179 \\
T5 & F.Terra firme & 71 & 3.930 & 0.921 & 2729.05 & 39.743 \\
T8 & F.Terra firme & 72 & 3.883 & 0.908 & 1889.09 & 25.935 \\
T2 & Mata Ciliar & 56 & 3.617 & 0.899 & 1957.55 & 49.681 \\
T4 & Mata Ciliar & 55 & 3.449 & 0.861 & 1559.53 & 44.359 \\
T6 & Mata Ciliar & 58 & 3.471 & 0.855 & 1884.84 & 46.704 \\
T7 & Mata Ciliar & 53 & 3.464 & 0.872 & 2125.70 & 27.584 \\
T10 & Mata Ciliar & 67 & 3.692 & 0.878 & 2868.74 & 51.009 \\
T3 & Mata alagada & 30 & 2.872 & 0.844 & 3604.49 & 23.506 \\
T9 & Mata alagada & 23 & 2.510 & 0.718 & 4299.98 & 18.884 \\
\hline
\end{tabular}

florestas amazônicas, tais como as florestas de terra firme de Manaus, com $H^{\prime}=4,76$ (Prance et al., 1976), as florestas da Serra dos Carajás no Pará, com $H^{\prime}=3,98$ (Morellato \& Rosa, 1991) e os igapós do Rio Negro, com $H^{\prime}=4,36$ (Keel \& Prance, 1979). As diversidades aqui encontradas são comparáveis a algumas florestas em áreas de tensão ecológica, como as ilhas de mata na região de contato floresta-savana em Roraima, com $\mathrm{H}^{\prime}=3,10 ; 4,27 ; 4,83$; e 3,75 (Silva, 1993).

$\mathrm{O}$ indice de diversidade revela aspectos da estrutura de uma comunidade e pode mostrar padrões gerais quando se comparam comunidades que diferem na composição de espécies (Bulla, 1994). Um baixo valor no indice de diversidade indica que uma ou poucas espécies são altamente abundantes e um alto valor indica que muitas espécies são igualmente abundantes na comunidade (Wilson et al., 1996a). Os resultados fitossociológicos dos transectos da mata alagada confirmam a dominância de poucas espécies nas comunidades estudadas.

\section{Estrutura Fitossociológica}

Nos transectos de floresta de terra firme (T1, T5 e T8) as dez principais espécies somam 44, 48 e $42 \%$ do valor total do IVI, respectivamente (Tabs. $4,5,6$ ). O indice de importância foi bastante 
Tabela 3. Riqueza (S), Diversidade ( $\left.\mathrm{H}^{\prime}\right)$, Equitabilidade (E), Estimativa da densidade total (DT - individuos/ha) e Estimativa da área basal total ( $\mathrm{AB}$ - área basal/ha) das árvores $(\mathrm{CAP} \geq 10 \mathrm{~cm})$ inventariadas em 10 transectos de ponto quadrante ( 50 pontos) amostrados no Rio Comemoração, Município de Pimenta Bueno, Rondônia.

\begin{tabular}{ccccccc}
\hline Transecto & Fisionomia & S & $H$ & E & DT & AB(m2) \\
\hline T1 & F.Terra firme & 71 & 3.888 & 0.912 & 2140.29 & 38.179 \\
T5 & F. Terra firme & 71 & 3.930 & 0.921 & 2729.05 & 39.743 \\
T8 & F.Terra firme & 72 & 3.883 & 0.908 & 1889.09 & 25.935 \\
T2 & Mata Ciliar & 56 & 3.617 & 0.899 & 1957.55 & 49.681 \\
T4 & Mata Cillar & 55 & 3.449 & 0.861 & 1559.53 & 44.359 \\
T6 & Mata Ciliar & 58 & 3.471 & 0.855 & 1884.84 & 46.704 \\
T7 & Mata Ciliar & 53 & 3.464 & 0.872 & 2125.70 & 27.584 \\
T10 & Mata Ciliar & 67 & 3.692 & 0.878 & 2868.74 & 51.009 \\
T3 & Mata alagada & 30 & 2.872 & 0.844 & 3604.49 & 23.506 \\
T9 & Mata alagada & 23 & 2.510 & 0.718 & 4299.98 & 18.884 \\
\hline
\end{tabular}

influenciado pela dominância relativa das espécies. Espécies com apenas um indivíduos, mas com grande área basal, ocuparam os primeiros lugares na ordem de importância, como: Maquira guianensis (IVI=10,61) no transecto T1; Macrolobium acaciefolium (IVI=37,1) no transecto T5; Qualea paraensis (IVI $=10,5$ ) no transecto T8 (Tabs. 4, 5, 6).

Nos transectos de mata ciliar (T2, T4, T6, T7 e T10) as dez principais espécies somam $60,63,63$, 58 e $52 \%$ do valor total do IVI, respectivamente (Tabs. 7, 8, 9, 10, 11). No transecto T2, as dez principais espécies somam $48 \%$ dos individuos amostrados e apenas Maquira guianensis (IVI $=62,3$ ) soma $47 \%$ da dominância relativa (Tab. 7). No transecto $\mathrm{T} 4$, as dez principais espécies somam $55 \%$ dos individuos e apenas Maquira guianensis $(\mathrm{IVI}=43,2)$ e Macrolobium acaciefolium (IVI=28,8) somam $54 \%$ da dominância relativa (Tab. 8). No transecto $\mathrm{T} 6$, as dez principais espécies somam $57 \%$ dos indivíduos amostrados e apenas Maquira guianensis (IVI=66,6) soma $43 \%$ da dominância relativa (Tab. 9). No transecto $\mathrm{T} 7$, as dez principais espécies somam $55 \%$ dos indivíduos amostrados e Zygia latifolia (IVI $=28,7$ ), Couratari tenuicarpa (IVI=20,2), Mauritia flexuosa (IVI=19,4) e Maquira guianensis (IVI $=16,1)$ somam $49 \%$ da 
Tabela 4. Parâmetros fitossociológicos das espécies inventariadas no transecto T1 (Floresta Terra firme).

\begin{tabular}{|c|c|c|c|c|c|}
\hline Espécie & $n$ & $\operatorname{Dr}(\%)$ & $\operatorname{Fr}(\%)$ & $\operatorname{Dor}(\%)$ & IVI \\
\hline Qualea paraensis & 4 & 2,0 & 2,20 & 29,59 & 33,79 \\
\hline Protium apiculatum & 22 & 11,0 & 9,34 & 4,09 & 24,43 \\
\hline Tachigalia paniculata & 12 & 6,0 & 4,95 & 3,65 & 14,59 \\
\hline Pseudomedia laevigata & 8 & 4,0 & 4,40 & 3,40 & 11,80 \\
\hline Maquira guianensis & 1 & 0,5 & 0,55 & 9,56 & 10,61 \\
\hline Prieurella prieurii & 8 & 4,0 & 4,40 & 0,70 & 9,10 \\
\hline Micropholis venulosa & 6 & 3,0 & 2,20 & 2,49 & 7,69 \\
\hline Miconia burchelli & 5 & 2,5 & 2,20 & 2,48 & 7,18 \\
\hline Ocotea canaliculata & 4 & 2,0 & 2,20 & 2,71 & 6,91 \\
\hline Eschweilera coriacea & 5 & 2,5 & 2,75 & 1,59 & 6,84 \\
\hline Euterpe precatoria & 6 & 3,0 & 2,75 & 1,05 & 6,80 \\
\hline Qualea acuminata & 6 & 3,0 & 3,30 & 0,49 & 6,78 \\
\hline Dialium guianense & 6 & 3,0 & 2,75 & 0,89 & 6,64 \\
\hline Protium trifoliolatum & 4 & 2,0 & 2,20 & 1,98 & 6,18 \\
\hline Oenocarpus bataua & 3 & 1,5 & 1,65 & 2,86 & 6,01 \\
\hline Gilibertia palustris & 5 & 2,5 & 2,75 & 0,55 & 5,80 \\
\hline Parkia panurensis & 2 & 1,0 & 1,10 & 3,55 & 5,65 \\
\hline Heisteria barbata & 4 & 2,0 & 2,20 & 0,89 & 5,08 \\
\hline Xylopia cf. calophylla & 3 & 1,5 & 1,65 & 1,82 & 4,97 \\
\hline Tachigalia myrmecophylla & 2 & 1,0 & 1,10 & 2,40 & 4,50 \\
\hline Tovomita brevistaminea & 4 & 20 & 1,65 & 0,83 & 4,48 \\
\hline Hebepetalum humirifolium & 3 & 1,5 & 1,65 & 0,83 & 3,97 \\
\hline Guatteria foliosa & 3 & 1,5 & 1,65 & 0,82 & 3,97 \\
\hline Hevea brasiliensis & 3 & 1,5 & 1,65 & 0,70 & 3,85 \\
\hline Aspidosperma tomentosum & 2 & 1,0 & 1,10 & 1,74 & 3,84 \\
\hline Iryanthera juruensis & 3 & 1,5 & 1,65 & 0,61 & 3,76 \\
\hline Tachigalia alba & 4 & 2,0 & 1,10 & 0,53 & 3,63 \\
\hline Calophyllum brasiliense & 3 & 1,5 & 1,65 & 0,39 & 3,54 \\
\hline Anacardium giganteum & 1 & 0,5 & 0,55 & 2,37 & 3,42 \\
\hline Mouriri cf. callocarpa & 3 & 1,5 & 1,65 & 0,23 & 3,38 \\
\hline Matayba arborescens & 3 & 1,5 & 1,65 & 0,19 & 3,34 \\
\hline Aspidosperma cf. verruculosum & 2 & 1,0 & 1,10 & 1,01 & 3,11 \\
\hline Inga falcistipula & 2 & 1,0 & 1,10 & 0,60 & 2,70 \\
\hline Amanoa guianensis & 1 & 0,5 & 0,55 & 1,65 & 2,70 \\
\hline Faramea longifolia & 2 & 1,0 & 1,10 & 0,35 & 2,45 \\
\hline Licania membranacea & 2 & 1,0 & 1,10 & 0,29 & 2,39 \\
\hline Guatteria duckeana & 2 & 1,0 & 1,10 & 0,24 & 2,34 \\
\hline Hirtella excelsa & 2 & 1,0 & 1,10 & 0,17 & 2,27 \\
\hline Neea ovalifolia & 2 & 1,0 & 1,10 & 0,14 & 2,24 \\
\hline Virola theiodora & 2 & 1,0 & 1,10 & 0,14 & 2,24 \\
\hline Toulicia cf. patentinervis & 1 & 0,5 & 0,55 & 1,19 & 2,24 \\
\hline Hirtella bicornis & 2 & 1,0 & 1,10 & 0,14 & 2,24 \\
\hline Miconia chrysophylla & 2 & 1,0 & 1,10 & 0,13 & 2,22 \\
\hline Virola sebifera & 1 & 0,5 & 0,55 & 1,06 & 2,11 \\
\hline Sloanea garckeana & 1 & 0,5 & 0,55 & 1,03 & 2,08 \\
\hline Outras 26 espécies & 28 & 14,0 & 14,29 & 5,86 & 34,15 \\
\hline Total & 200 & 100 & 100 & 100 & 300 \\
\hline
\end{tabular}


Tabela 5. Parâmetros fitossociológicos das espécies inventariadas no transecto T5 (Floresta Terra firme).

\begin{tabular}{|c|c|c|c|c|c|}
\hline Espécie & $\mathrm{n}$ & $\operatorname{Dr}(\%)$ & $\operatorname{Fr}(\%)$ & Dor(\%) & IVI \\
\hline Macrolobium acaciefolium & 1 & 0,5 & 0,52 & 36,05 & 37.07 \\
\hline Tachigalia myrmecophilla & 9 & 4,5 & 4,69 & 7,40 & 16.58 \\
\hline Amanoa guianensis & 13 & 6,5 & 5,73 & 4,20 & 16.43 \\
\hline Dialium guianense & 9 & 4,5 & 4,69 & 3,27 & 12.46 \\
\hline Protium heptaphyllum & 11 & 5,5 & 5,73 & 0,39 & 11.61 \\
\hline Licania membranacea & 8 & 4,0 & 4,17 & 3,34 & 11,51 \\
\hline Anaxagorea acuminata & 11 & 5,5 & 5,21 & 0,76 & 11,47 \\
\hline Parkia panurensis & 6 & 3,0 & 2,60 & 4,27 & 9,87 \\
\hline Euterpe precatoria & 8 & 4,0 & 4,17 & 1,62 & 9,79 \\
\hline Rheedia brasiliensis & 5 & 2,5 & 2,08 & 3,06 & 7,65 \\
\hline Gilibertia palustris & 6 & 3,0 & 2,60 & 1,62 & 7,23 \\
\hline Leonia glycycarpa & 6 & 3,0 & 2,60 & 0,59 & 6,20 \\
\hline Tovomita cf. speciosa & 5 & 2,5 & 2,60 & 0,83 & 5,94 \\
\hline Tachigalia alba & 5 & 2,5 & 2,08 & 1,24 & 5,83 \\
\hline Tovomita brevistaminea & 4 & 2,0 & 2,08 & 1,28 & 5,36 \\
\hline Neea ovalifolia & 3 & 1,5 & 1,56 & 2,10 & 5,16 \\
\hline Oenocarpus bataua & 2 & 1,0 & 1,04 & 2,82 & 4,87 \\
\hline Hymenolobium nitidum & 3 & 1,5 & 1,56 & 1,11 & 4,17 \\
\hline Protium trifoliolatum & 2 & 1,0 & 1,04 & 1,97 & 4,01 \\
\hline Qualea dinizii & 1 & 0,5 & 0,52 & 2,83 & 3,85 \\
\hline Licania heteromorpha & 3 & 1,5 & 1,56 & 0,63 & 3,69 \\
\hline Myrtiluma eugenifolia & 3 & 1,5 & 1,56 & 0,56 & 3,62 \\
\hline Micropholis cyrtobotria & 3 & 1,5 & 1,56 & 0,49 & 3,55 \\
\hline Xylopia cf. calophylla & 3 & 1,5 & 1,56 & 0,42 & 3,48 \\
\hline Calyptranthus creba & 3 & 1,5 & 1,56 & 0,42 & 3,48 \\
\hline Hirtella racemosa & 3 & 1,5 & 1,56 & 0,39 & 3,45 \\
\hline Matayba arborescens & 3 & 1,5 & 1,56 & 0,39 & 3,45 \\
\hline Pseudomedia murure & 3 & 1,5 & 1,56 & 0,39 & 3,45 \\
\hline Anacardium giganteum & 2 & 1,0 & 1,04 & 1,42 & 3,46 \\
\hline Ouratea cf. paraensis & 3 & 1,5 & 1,56 & 0,25 & 3,31 \\
\hline Hirtella bicornis & 3 & 1,5 & 1,56 & 0,18 & 3,24 \\
\hline Pachira aquatica & 1 & 0,5 & 0,52 & 2,16 & 3,18 \\
\hline Qualea paraensis & 2 & 1,0 & 1,04 & 1,00 & 3,05 \\
\hline Guatteria duckeana & 2 & 1,0 & 1,04 & 0,76 & 2,81 \\
\hline Faramea longifolia & 2 & 1,0 & 1,04 & 0,45 & 2,50 \\
\hline Micropholis venulosa & 2 & 1,0 & 1,04 & 0,45 & 2,50 \\
\hline Astronium lecointei & 2 & 1,0 & 1,04 & 0,39 & 2,43 \\
\hline Iryanthera juruensis & 2 & 1,0 & 1,04 & 0,39 & 2,43 \\
\hline Tachigalia paniculata & 2 & 1,0 & 1,04 & 0,32 & 2,36 \\
\hline Prieurella prieurii & 2 & 1,0 & 1,04 & 0,28 & 2,32 \\
\hline Inga nitida & 2 & 1,0 & 1,04 & 0,18 & 2,22 \\
\hline Ilex parviflora & 1 & 0,5 & 0,52 & 1,20 & 2,22 \\
\hline Tococa guianensis & 2 & 1,0 & 1,04 & 0,15 & 2,19 \\
\hline Brosimum guianense & 1 & 0,5 & 0,52 & 1,09 & 2,11 \\
\hline Theobroma speciosum & 1 & 0,5 & 0,52 & 0,42 & 1,44 \\
\hline Outras 26 espécies & 26 & 13,0 & 13,54 & 4,51 & 31,05 \\
\hline Total & 200 & 100 & 100 & 100 & 300 \\
\hline
\end{tabular}


Tabela 6. Parâmetros fitossociológicos das espécies inventariadas no transecto T8 (Floresta Terra firme).

\begin{tabular}{|c|c|c|c|c|c|}
\hline Espécie & $\mathrm{n}$ & $\operatorname{Dr}(\%)$ & $\operatorname{Fr}(\%)$ & Dor(\%) & IVI \\
\hline Hirtella bicornis & 16 & 8,0 & 7,41 & 1,19 & 16,59 \\
\hline Protium heptaphyllum & 7 & 3,5 & 3,70 & 7,82 & 15,02 \\
\hline Swartzia arborescens & 9 & 4,5 & 4,23 & 4,85 & 13,58 \\
\hline Ocotea canaliculata & 7 & 3,5 & 3,70 & 6,36 & 13,56 \\
\hline Protium trifoliolatum & 12 & 6,0 & 5,29 & 2,03 & 13,32 \\
\hline Dialium guianense & 4 & 2,0 & 1,59 & 7,69 & 11,28 \\
\hline Nectandra amazonum & 10 & 5,0 & 4,76 & 1,29 & 11,06 \\
\hline Matayba arborescens & 9 & 4,5 & 4,76 & 1,71 & 10,97 \\
\hline Qualea paraensis & 1 & 0,5 & 0,53 & 9,50 & 10,52 \\
\hline Micropholis venulosa & 6 & 3,0 & 3,17 & 2,87 & 9,05 \\
\hline Mezilaurus aff. subcordata & 7 & 3,5 & 2,65 & 1,87 & 8,02 \\
\hline Iryanthera sagotiana & 6 & 3,0 & 3,17 & 0,88 & 7,06 \\
\hline Qualea acuminata & 6 & 3,0 & 3,17 & 0,60 & 6,77 \\
\hline Miconia chrysophylla & 6 & 3,0 & 2,65 & 1,01 & 6,66 \\
\hline Casearia javitensis & 2 & 1,0 & 1,06 & 4,50 & 6,56 \\
\hline Sloanea garckeana & 3 & 1,5 & 1,59 & 3,04 & 6,13 \\
\hline Myrtiluma eugenifolia & 5 & 2,5 & 2,65 & 0,92 & 6,07 \\
\hline Pseudomedia murure & 4 & 2,0 & 2,12 & 1,44 & 5,56 \\
\hline Calophyllum brasiliense & 4 & 2,0 & 2,12 & 1,30 & 5,42 \\
\hline Diplotropis brasiliensis & 1 & 0,5 & 0,53 & 4,04 & 5,06 \\
\hline Prieurella prieurii & 3 & 1,5 & 1,59 & 1,65 & 4,74 \\
\hline Mezilaurus synandra & 1 & 0,5 & 0,53 & 3,44 & 4,47 \\
\hline Gilibertia palustris & 2 & 1,0 & 1,06 & 2,33 & 4,39 \\
\hline Toulicia cf. patentinervis & 3 & 1,5 & 1,59 & 1,22 & 4,31 \\
\hline Pseudomedia laevigata & 2 & 1,0 & 1,06 & 2,16 & 4,22 \\
\hline Pterocarpus rohrii & 2 & 1,0 & 1,06 & 1,81 & 3,87 \\
\hline Astronium lecointei & 2 & 1,0 & 1,06 & 1,78 & 3,83 \\
\hline Euterpe precatoria & 3 & 1,5 & 1,59 & 0,52 & 3,61 \\
\hline Miconia burchelli & 3 & 1,5 & 1,59 & 0,11 & 3,20 \\
\hline Licania heteromorpha & 2 & 1,0 & 1,06 & 1,00 & 3,06 \\
\hline Mouriri cf. callocarpa & 2 & 1,0 & 1,06 & 0,94 & 3,00 \\
\hline Licania membranacea & 3 & 1,5 & 1,06 & 0,41 & 2,96 \\
\hline Micropholis guianensis & 1 & 0,5 & 0,53 & 1,76 & 2,79 \\
\hline Inga nitida & 1 & 0,5 & 0,53 & 1,67 & 2,70 \\
\hline Diospyros duckei & 2 & 1,0 & 1,06 & 0,56 & 2,62 \\
\hline Mezilaurus itauba & 2 & 1,0 & 1,06 & 0,42 & 2,48 \\
\hline Pouteria caimito & 2 & 1,0 & 1,06 & 0,24 & 2,30 \\
\hline Tachigalia myrmecophilla & 2 & 1,0 & 1,06 & 1,30 & 3,36 \\
\hline Sclerolobium aureum & 2 & 1,0 & 1,06 & 0,19 & 2,25 \\
\hline Zanthoxylum sprucei & 2 & 1,0 & 1,06 & 0,10 & 2,16 \\
\hline Duguettia calycina & 2 & 1,0 & 1,06 & 0,08 & 2,14 \\
\hline Aspidosperma tomentosum & 1 & 0,5 & 0,53 & 1,08 & 2,11 \\
\hline Pouroma bicolor & 1 & 0,5 & 0,53 & 1,04 & 2,07 \\
\hline Salacia sp. & 1 & 0,5 & 0,53 & 1,01 & 2,04 \\
\hline Anacardium giganteum & 1 & 0,5 & 0,53 & 0,97 & 2,00 \\
\hline Outras 27 espécies & 27 & 13,5 & 14,29 & 7,28 & 35,06 \\
\hline Total & 200 & 100 & 100 & 100 & 300 \\
\hline
\end{tabular}


Tabela 7. Parâmetros fitossociológicos das espécies inventariadas no transecto T2 (Mata Ciliar).

\begin{tabular}{|c|c|c|c|c|c|}
\hline Espécie & $n$ & $\operatorname{Dr}(\%)$ & $\operatorname{Fr}(\%)$ & $\operatorname{Dor}(\%)$ & $\mid \mathrm{VI}$ \\
\hline Maquira guianensis & 17 & 8,5 & 6,56 & 47,24 & 62,30 \\
\hline Nectandra amazonum & 16 & 8,0 & 8,20 & 2,34 & 18,54 \\
\hline Couratari tenuicarpa & 7 & 3,5 & 3,28 & 8,90 & 15,68 \\
\hline Amanoa guianensis & 13 & 6,5 & 6,56 & 2,50 & 15,56 \\
\hline Macrolobium acaciefolium & 5 & 2,5 & 2,73 & 9,38 & 14,61 \\
\hline Tachigalia myrmecophilla & 9 & 4,5 & 4,92 & 3,33 & 12,75 \\
\hline Pithecelobium Iatifolium & 7 & 3,5 & 3,83 & 3,09 & 10,42 \\
\hline Hevea brasiliensis & 6 & 3,0 & 3,28 & 3,92 & 10,20 \\
\hline Protium apiculatum & 9 & 4,5 & 4,92 & 0,75 & 10,17 \\
\hline Licania membranacea & 8 & 4,0 & 4,37 & 1,50 & 9,87 \\
\hline Leonia glycycarpa & 8 & 4,0 & 3,83 & 0,28 & 8,10 \\
\hline Inga cayennensis & 7 & 3,5 & 2,19 & 1,12 & 6,81 \\
\hline Zygia latifolia & 7 & 3,5 & 2,19 & 0,53 & 6,22 \\
\hline Calyptranthus creba & 5 & 2,5 & 2,73 & 0,67 & 5,90 \\
\hline Dulacia candida & 5 & 2,5 & 2,73 & 0,65 & 5,88 \\
\hline Euterpe precatoria & 5 & 2,5 & 2,73 & 0,30 & 5,53 \\
\hline Miconia burchelli & 3 & 1,5 & 1,64 & 1,50 & 4,64 \\
\hline Iryanthera juruensis & 4 & 2,0 & 2,19 & 0,33 & 4,52 \\
\hline Guatteria duckeana & 3 & 1,5 & 1,64 & 0,43 & 3,57 \\
\hline Brosimum guianense & 3 & 1,5 & 1,64 & 0,33 & 3,47 \\
\hline Genipa americana & 3 & 1,5 & 1,09 & 0,73 & 3,32 \\
\hline Prieurella prieurii & 3 & 1,5 & 1,64 & 0,18 & 3,32 \\
\hline Inga ingoides & 2 & 1,0 & 1,09 & 0,85 & 2,94 \\
\hline Licania apetala & 2 & 1,0 & 1,09 & 0,81 & 2,90 \\
\hline Eschweilera coriacea & 2 & 1,0 & 1,09 & 0,75 & 2,84 \\
\hline Vismia macrophylla & 2 & 1,0 & 1,09 & 0,73 & 2,82 \\
\hline Ocotea canaliculata & 2 & 1,0 & 1,09 & 0,65 & 2,74 \\
\hline Xylopia cf. calophylla & 2 & 1,0 & 1,09 & 0,47 & 2,57 \\
\hline Theobroma speciosum & 2 & 1,0 & 1,09 & 0,41 & 2,51 \\
\hline Mauritia flexuosa & 1 & 0,5 & 0,55 & 1,44 & 2,49 \\
\hline Byrsonima stipulacea & 2 & 1,0 & 1,09 & 0,22 & 2,31 \\
\hline Micropholis venulosa & 2 & 1,0 & 1,09 & 0,16 & 2,25 \\
\hline Rinorea guianensis & 2 & 1,0 & 1,09 & 0,08 & 2,17 \\
\hline Licania octandra & 2 & 1,0 & 1,09 & 0,06 & 2,15 \\
\hline Inga cf. cinnanomea & 2 & 1,0 & 1,09 & 0,04 & 2,13 \\
\hline Croton sp. & 1 & 0,5 & 0,55 & 0,91 & 1,95 \\
\hline Mouriri apiranga & 1 & 0,5 & 0,55 & 0,60 & 1,65 \\
\hline Mouriri myrtifolia & 2 & 1,0 & 0,55 & 0,08 & 1,63 \\
\hline Salacia sp. & 1 & 0,5 & 0,55 & 0,39 & 1,44 \\
\hline Calophyllum brasiliense & 1 & 0,5 & 0,55 & 0,25 & 1,30 \\
\hline Minquartia guianensis & 1 & 0,5 & 0,55 & 0,25 & 1,30 \\
\hline Sorocea guilleminiana & 1 & 0,5 & 0,55 & 0,11 & 1,16 \\
\hline Micropholis cyrtobotria & 1 & 0,5 & 0,55 & 0,10 & 1,14 \\
\hline Anaxagorea acuminata & 1 & 0,5 & 0,55 & 0,10 & 1,14 \\
\hline Dialium guianense & 1 & 0,5 & 0,55 & 0,08 & 1,13 \\
\hline Outras 11 espécies & 11 & 5,5 & 6,01 & 0,45 & 11,96 \\
\hline Total & 200 & 100 & 100 & 100 & 300 \\
\hline
\end{tabular}


Tabela 8. Parâmetros fitossociológicos das espécies inventariadas no transecto T4 (Mata Ciliar).

\begin{tabular}{|c|c|c|c|c|c|}
\hline Espécie & $n$ & $\operatorname{Dr}(\%)$ & $\operatorname{Fr}(\%)$ & Dor(\%) & $\mid \mathrm{VI}$ \\
\hline Maquira guianensis & 15 & 7,5 & 7,26 & 28,42 & 43,18 \\
\hline Macrolobium acaciefolium & 3 & 1,5 & 1,68 & 25,67 & 28,85 \\
\hline Amanoa guianensis & 18 & 9,0 & 9,50 & 9,19 & 27,68 \\
\hline Licaria armeniaca & 29 & 14,5 & 11,17 & 1,91 & 27,59 \\
\hline Iriartella setigera & 20 & 10,0 & 8,38 & 2,82 & 21,2 \\
\hline Hevea brasiliensis & 5 & 2,5 & 2,79 & 7,28 & 12.57 \\
\hline Leonia glycycarpa & 7 & 3,5 & 3,91 & 1,32 & 8,73 \\
\hline Mouriri cf. callocarpa & 6 & 3,0 & 3,35 & 0,61 & 6,97 \\
\hline Zygia latifolia & 6 & 3,0 & 2,79 & 0,90 & 6,69 \\
\hline Coussapoa angustifolia & 1 & 0,5 & 0,56 & 5,60 & 6,65 \\
\hline Licania membranacea & 2 & 1,0 & 1,12 & 3,76 & 5,88 \\
\hline Duguettia calycina & 4 & 2,0 & 2,23 & 0,28 & 4,51 \\
\hline Guatteria duckeana & 4 & 2,0 & 2,23 & 0,23 & 4,46 \\
\hline Iryanthera juruensis & 4 & 2,0 & 2,23 & 0,15 & 4,38 \\
\hline Licania gardneri & 4 & 2,0 & 1,68 & 0,41 & 4,09 \\
\hline Euterpe precatoria & 3 & 1,5 & 1,68 & 0,81 & 3,99 \\
\hline Pterocarpus rohii & 3 & 1,5 & 1,68 & 0,74 & 3,92 \\
\hline Protium apiculatum & 3 & 1,5 & 1,68 & 0,70 & 3,88 \\
\hline Micropholis venulosa & 3 & 1,5 & 1,12 & 1,07 & 3,69 \\
\hline Tachigalia myrmecophilla & 3 & 1,5 & 1,68 & 0,20 & 3,37 \\
\hline Inga nitida & 3 & 1,5 & 1,68 & 0,18 & 3,36 \\
\hline Pseudomedia murure & 3 & 1,5 & 1,68 & 0,16 & 3,34 \\
\hline Vismia macrophylla & 3 & 1,5 & 1,68 & 0,14 & 3,32 \\
\hline Theobroma speciosum & 3 & 1,5 & 1,68 & 0,13 & 3,31 \\
\hline Couratari tenuicarpa & 2 & 1,0 & 1,12 & 1,14 & 3,26 \\
\hline Tococa guiaensis & 3 & 1,5 & 1,68 & 0,05 & 3,23 \\
\hline Anacardium giganteum & 2 & 1,0 & 1,12 & 0,56 & 2,67 \\
\hline Hirtella racemosa & 2 & 1,0 & 1,12 & 0,41 & 2,53 \\
\hline Mauritia flexuosa & 1 & 0,5 & 0,56 & 1,46 & 2,51 \\
\hline Inga velutina & 2 & 1,0 & 1,12 & 0,39 & 2,50 \\
\hline Sterculia speciosa & 2 & 1,0 & 1,12 & 0,28 & 2,39 \\
\hline Rheedia macrophylla & 2 & 1,0 & 1,12 & 0,23 & 2,35 \\
\hline Pseudomedia laevigata & 2 & 1,0 & 1,12 & 0,12 & 2,24 \\
\hline Gilibertia palustris & 2 & 1,0 & 1,12 & 0,11 & 2,23 \\
\hline Brosimum guianense & 2 & 1,0 & 1,12 & 0,09 & 2,21 \\
\hline Miconia burchelli & 2 & 1,0 & 1,12 & 0,06 & 2,18 \\
\hline Dialium guianense & 2 & 1,0 & 1,12 & 0,05 & 2,17 \\
\hline Licania apetala & 1 & 0,5 & 0,56 & 0,57 & 1,63 \\
\hline Pouteria caimito & 2 & 1,0 & 0,56 & 0,05 & 1,61 \\
\hline Brosimum acutifolium & 1 & 0,5 & 0,56 & 0,35 & 1,41 \\
\hline Luehea sp. & 1 & 0,5 & 0,56 & 0,30 & 1,35 \\
\hline Hirtella bicornis & 1 & 0,5 & 0,56 & 0,17 & 1,23 \\
\hline Sparathanthelium tupiniquinorum & 1 & 0,5 & 0,56 & 0,17 & 1,23 \\
\hline Ouratea cf. paraensis & 1 & 0,5 & 0,56 & 0,13 & 1,19 \\
\hline Micropholis guianensis & 1 & 0,5 & 0,56 & 0,13 & 1,18 \\
\hline Outras 10 espécies & 10 & 5,0 & 5,59 & 0,50 & 11,08 \\
\hline Total & 200 & 100 & 100 & 100 & 300 \\
\hline
\end{tabular}


Tabela 9. Parâmetros fitossociológicos das espécies inventariadas no transecto T6 (Mata Ciliar).

\begin{tabular}{|c|c|c|c|c|c|}
\hline Espécie & $n$ & $\operatorname{Dr}(\%)$ & $\operatorname{Fr}(\%)$ & Dor(\%) & IVI \\
\hline Maquira guianensis & 26 & 13,0 & 10,37 & 43,22 & 66,59 \\
\hline Amanoa guianensis & 15 & 7,5 & 6,71 & 9,78 & 23,99 \\
\hline Zygia latifolia & 19 & 9,5 & 9,15 & 2,85 & 21,49 \\
\hline Sloanea garckeana & 9 & 4,5 & 4,88 & 4,94 & 14,32 \\
\hline Nectandra amazonum & 12 & 6,0 & 5,49 & 0,57 & 12,05 \\
\hline Xylopia nitida & 10 & 5,0 & 5,49 & 1,47 & 11,96 \\
\hline Genipa americana & 6 & 3,0 & 3,05 & 5,23 & 11,28 \\
\hline Eschwelera coriacea & 5 & 2,5 & 2,44 & 5,83 & 10,77 \\
\hline Macrolobium acaciefolium & 7 & 3,5 & 3,05 & 4,04 & 10,58 \\
\hline Calyptranthus creba & 6 & 3,0 & 3,05 & 0,79 & 6,84 \\
\hline Pterocarpus rohrii & 5 & 2,5 & 2,44 & 1,70 & 6,63 \\
\hline Indet.1 & 6 & 3,0 & 3,05 & 0,57 & 6,61 \\
\hline Panopsis rubescens & 4 & 2,0 & 2,44 & 1,45 & 5,89 \\
\hline Cecropia concolor & 4 & 2,0 & 2,44 & 1,43 & 5,87 \\
\hline Croton sp. & 5 & 2,5 & 1,22 & 2,08 & 5,80 \\
\hline Hevea brasiliensis & 2 & 1,0 & 1,22 & 3,53 & 5,75 \\
\hline Gilibertia palustris & 3 & 1,5 & 1,83 & 2,30 & 5,63 \\
\hline Siparuna guianensis & 6 & 3,0 & 2,44 & 0,16 & 5,60 \\
\hline Inga nitida & 3 & 1,5 & 1,83 & 0,59 & 3,91 \\
\hline Mouriri cf. callocarpa & 3 & 1,5 & 1,83 & 0,38 & 3,71 \\
\hline Hirtella racemosa & 2 & 1,0 & 1,22 & 0,40 & 2,62 \\
\hline Casearia sylvestris & 2 & 1,0 & 0,61 & 1,01 & 2,62 \\
\hline Toulicia cf. patentinervis & 2 & 1,0 & 1,22 & 0,38 & 2,60 \\
\hline Zigia cauliflora & 2 & 1,0 & 1,22 & 0,14 & 2,36 \\
\hline Minquartia guianensis & 2 & 1,0 & 1,22 & 0,12 & 2,34 \\
\hline Mabea fistulifera & 2 & 1,0 & 0,61 & 0,22 & 1,83 \\
\hline Miconia burchelli & 1 & 0,5 & 0,61 & 0,62 & 1,73 \\
\hline Perebea mollis & 1 & 0,5 & 0,61 & 0,52 & 1,63 \\
\hline Brosimum guianense & 1 & 0,5 & 0,61 & 0,47 & 1,58 \\
\hline Euterpe precatoria & 1 & 0,5 & 0,61 & 0,39 & 1,50 \\
\hline Pouteria caimito & 1 & 0,5 & 0,61 & 0,37 & 1,48 \\
\hline Sorocea guilleminiana & 1 & 0,5 & 0,61 & 0,30 & 1,41 \\
\hline Licania apetala & 1 & 0,5 & 0,61 & 0,30 & 1,41 \\
\hline Byrsonima stipulacea & 1 & 0,5 & 0,61 & 0,27 & 1,38 \\
\hline Copaifera reticulata & 1 & 0,5 & 0,61 & 0,20 & 1,31 \\
\hline Coussapoa angustifolia & 1 & 0,5 & 0,61 & 0,19 & 1,30 \\
\hline Pera distichophylla & 1 & 0,5 & 0,61 & 0,15 & 1,26 \\
\hline Indet.2 & 1 & 0,5 & 0,61 & 0,13 & 1,24 \\
\hline Prieurella prieurii & 1 & 0,5 & 0,61 & 0,10 & 1,21 \\
\hline Iriartella setigera & 1 & 0,5 & 0,61 & 0,10 & 1,21 \\
\hline Swartzia grandiflora & 1 & 0,5 & 0,61 & 0,08 & 1,19 \\
\hline Macrolobium sp. & 1 & 0,5 & 0,61 & 0,08 & 1,19 \\
\hline Ouratea cf. paraensis & 1 & 0,5 & 0,61 & 0,07 & 1,18 \\
\hline Symphonia globulifera & 1 & 0,5 & 0,61 & 0,07 & 1,18 \\
\hline Matayba arborescens & 1 & 0,5 & 0,61 & 0,05 & 1,16 \\
\hline Outras 13 espécies & 13 & 6,5 & 7,93 & 0,39 & 14,81 \\
\hline Total & 200 & 100 & 100 & 100 & 300 \\
\hline
\end{tabular}


Tabela 10. Parâmetros fitossociológicos das espécies inventariadas no transecto T7 (Mata Ciliar).

\begin{tabular}{|c|c|c|c|c|c|}
\hline Espécie & $n$ & $\operatorname{Dr}(\%)$ & $\operatorname{Fr}(\%)$ & Dor $(\%)$ & IVI \\
\hline Zygia latifolia & 24 & 12,0 & 6,47 & 10,22 & 28,69 \\
\hline Nectandra amazonum & 20 & 10,0 & 8,82 & 2,90 & 21,73 \\
\hline Couratari tenuicarpa & 5 & 2,5 & 2,94 & 14,77 & 20,21 \\
\hline Mauritia flexuosa & 4 & 2,0 & 2,35 & 15,05 & 19,40 \\
\hline Amanoa guianensis & 10 & 5,0 & 4,71 & 7,08 & 16,79 \\
\hline Maquira guianensis & 8 & 4,0 & 3,53 & 8,53 & 16,06 \\
\hline Mouriri cf. callocarpa & 11 & 5,5 & 5,29 & 2,86 & 13,65 \\
\hline Micropholis venulosa & 10 & 5,0 & 5,29 & 2,66 & 12,96 \\
\hline Licania membranacea & 9 & 4,5 & 5,29 & 2,85 & 12,65 \\
\hline Pterocarpus rohrii & 9 & 4,5 & 4,71 & 3,13 & 12,34 \\
\hline Eschweilera coriacea & 3 & 1,5 & 1,76 & 7,71 & 10,97 \\
\hline Panopsis rubescens & 5 & 2,5 & 2,94 & 3,18 & 8,62 \\
\hline Calyptranthus creba & 6 & 3,0 & 3,53 & 1,62 & 8,15 \\
\hline Tachigalia myrmecophilla & 6 & 3,0 & 3,53 & 1,04 & 7,57 \\
\hline Euterpe precatoria & 5 & 2,5 & 2,94 & 1,61 & 7,05 \\
\hline Hevea brasiliensis & 4 & 2,0 & 1,76 & 2,33 & 6,10 \\
\hline Iryanthera juruensis & 5 & 2,5 & 2,94 & 0,44 & 5,88 \\
\hline Protium apiculatum & 4 & 2,0 & 2,35 & 0,71 & 5,06 \\
\hline Macrolobium acaciefolium & 5 & 2,5 & 1,76 & 0,61 & 4,87 \\
\hline Nectandra lucida & 4 & 2,0 & 1,76 & 0,70 & 4,47 \\
\hline Xylopia cf. calophylla & 3 & 1,5 & 1,76 & 0,64 & 3,91 \\
\hline Dialium guianense & 3 & 1,5 & 1,76 & 0,55 & 3,82 \\
\hline Hirtella bicornis & 2 & 1,0 & 1,18 & 0,41 & 2,59 \\
\hline Miconia tomentosa & 2 & 1,0 & 1,18 & 0,23 & 2,41 \\
\hline Minquartia guianensis & 2 & 1,0 & 1,18 & 0,18 & 2,36 \\
\hline Pouteria caimito & 2 & 1,0 & 1,18 & 0,18 & 2,35 \\
\hline Licania gardneri & 2 & 1,0 & 1,18 & 0,16 & 2,34 \\
\hline Duguettia calycina & 2 & 1,0 & 1,18 & 0,10 & 2,28 \\
\hline Brosimum guianense & 1 & 0,5 & 0,59 & 1,10 & 2,19 \\
\hline Coussapoa angustifolia & 1 & 0,5 & 0,59 & 0,86 & 1,95 \\
\hline Guatteria poeppigiana & 1 & 0,5 & 0,59 & 0,80 & 1,89 \\
\hline Iriartella setigera & 1 & 0,5 & 0,59 & 0,68 & 1,77 \\
\hline Aspidosperma desmanthum & 1 & 0,5 & 0,59 & 0,57 & 1,66 \\
\hline Salacia sp. & 1 & 0,5 & 0,59 & 0,57 & 1,66 \\
\hline Protium trifoliolatum & 1 & 0,5 & 0,59 & 0,35 & 1,44 \\
\hline Prieurella prieurii & 1 & 0,5 & 0,59 & 0,31 & 1,40 \\
\hline Qualea acuminata & 1 & 0,5 & 0,59 & 0,31 & 1,40 \\
\hline Qualea paraensis & 1 & 0,5 & 0,59 & 0,31 & 1,40 \\
\hline Zigia cauliflora & 1 & 0,5 & 0,59 & 0,24 & 1,33 \\
\hline Matayba arborescens & 1 & 0,5 & 0,59 & 0,22 & 1,31 \\
\hline Byrsonima stipulacea & 1 & 0,5 & 0,59 & 0,21 & 1,30 \\
\hline Lacistema aggregatum & 1 & 0,5 & 0,59 & 0,19 & 1,28 \\
\hline Xylopia nitida & 1 & 0,5 & 0,59 & 0,19 & 1,28 \\
\hline Ficus trigona & 1 & 0,5 & 0,59 & 0,16 & 1,25 \\
\hline Sloanea garckeana & 1 & 0,5 & 0,59 & 0,09 & 1,18 \\
\hline Outras 8 espécies & 8 & 4,0 & 4,71 & 0,35 & 9,06 \\
\hline Total & 200 & 100 & 100 & 100 & 300 \\
\hline
\end{tabular}


Tabela 11. Parâmetros fitossociológicos das espécies inventariadas no transecto T10 (Mata Ciliar).

\begin{tabular}{|c|c|c|c|c|c|}
\hline Espécie & $\mathrm{n}$ & $\operatorname{Dr}(\%)$ & $\operatorname{Fr}(\%)$ & Dor(\%) & IVI \\
\hline Protium apiculatum & 17 & 8,5 & 8,11 & 7,02 & 23,63 \\
\hline Parkia panurensis & 3 & 1,5 & 1,62 & 18,39 & 21,51 \\
\hline Oenocarpus bataua & 12 & 6,0 & 5,95 & 9,52 & 21,47 \\
\hline Euterpe precatoria & 18 & 9,0 & 7,57 & 3,08 & 19,65 \\
\hline Mouriri cf. callocarpa & 14 & 7,0 & 7,57 & 4,44 & 19,01 \\
\hline Micropholis venulosa & 10 & 5,0 & 4,86 & 3,04 & 12,9 \\
\hline Protium trifoliolatum & 8 & 4,0 & 3,78 & 2,81 & 10,56 \\
\hline Tachigalia myrmecophilla & 9 & 4,5 & 4,32 & 0,68 & 9,51 \\
\hline Amanoa guianensis & 7 & 3,5 & 3,24 & 1,85 & 8,59 \\
\hline Macrolobium acaciefolium & 1 & 0,5 & 0,54 & 6,70 & 7,74 \\
\hline Qualea paraensis & 1 & 0,5 & 0,54 & 6,70 & 7,74 \\
\hline Salacia sp. & 4 & 2,0 & 2,16 & 3,53 & 7,69 \\
\hline Licania membranacea & 6 & 3,0 & 2,70 & 1,92 & 7,62 \\
\hline Luehea sp. & 2 & 1,0 & 1,08 & 4,94 & 7,02 \\
\hline Inga nitida & 6 & 3,0 & 2,70 & 0,48 & 6,18 \\
\hline Gilibertia palustris & 5 & 2,5 & 2,70 & 0,84 & 6,04 \\
\hline Jacaranda copaia & 2 & 1,0 & 1,08 & 2,96 & 5,04 \\
\hline Licania heteromorpha & 4 & 2,0 & 2,16 & 0,60 & 4,76 \\
\hline Myrtiluma eugenifolia & 3 & 1,5 & 1,62 & 1,53 & 4,65 \\
\hline Nectandra amazonum & 4 & 2,0 & 2,16 & 0,46 & 4,62 \\
\hline Simphonia globulifera & 1 & 0,5 & 0,54 & 3,55 & 4,59 \\
\hline Hevea brasiliensis & 3 & 1,5 & 1,62 & 1,11 & 4,24 \\
\hline Vataireopsis speciosa & 3 & 1,5 & 1,62 & 1,02 & 4,14 \\
\hline Minquartia guianensis & 3 & 1,5 & 1,62 & 0,86 & 3,98 \\
\hline Tovomita brevistaminea & 3 & 1,5 & 1,08 & 1,03 & 3,61 \\
\hline Guatteria duckeana & 2 & 1,0 & 1,08 & 0,91 & 2,99 \\
\hline Cariniana micrantha & 1 & 0,5 & 0,54 & 1,81 & 2,85 \\
\hline Hirtella racemosa & 2 & 1,0 & 1,08 & 0,73 & 2,82 \\
\hline Cheiloclinium cognata & 2 & 1,0 & 1,08 & 0,68 & 2,76 \\
\hline Hymenelobium sp. & 2 & 1,0 & 1,08 & 0,57 & 2,65 \\
\hline Casearia javitensis & 2 & 1,0 & 0,54 & 1,04 & 2,58 \\
\hline Ouratea cf. paraensis & 2 & 1,0 & 1,08 & 0,20 & 2,28 \\
\hline Matayba arborescens & 2 & 1,0 & 1,08 & 0,11 & 2,19 \\
\hline Tachigalia alba & 2 & 1,0 & 1,08 & 0,08 & 2,16 \\
\hline Tococa guianensis & 2 & 1,0 & 1,08 & 0,07 & 2,15 \\
\hline Anacardium giganteum & 1 & 0,5 & 0,54 & 0,89 & 1,93 \\
\hline Brosimum acutifolium & 1 & 0,5 & 0.54 & 0,54 & 1,58 \\
\hline Xylopia benthamii & 1 & 0,5 & 0,54 & 0,49 & 1,53 \\
\hline Xylopia calycina & 1 & 0,5 & 0,54 & 0,43 & 1,47 \\
\hline Pouroma bicolor & 1 & 0,5 & 0,54 & 0,27 & 1,31 \\
\hline Pseudomedia murure & 1 & 0,5 & 0,54 & 0,26 & 1,30 \\
\hline Brosimum potabile & 1 & 0,5 & 0,54 & 0,24 & 1,28 \\
\hline Diallium guianense & 1 & 0,5 & 0,54 & 0,16 & 1,20 \\
\hline Eschweilera coriacea & 1 & 0,5 & 0,54 & 0,16 & 1,20 \\
\hline Myrciaria floribunda & 1 & 0,5 & 0,54 & 0,16 & 1,20 \\
\hline Outras 22 espécies & 22 & 11,0 & 11,89 & 1,13 & 24,02 \\
\hline Total & 200 & 100 & 100 & 100 & 300 \\
\hline
\end{tabular}


dominância relativa (Tab. 10). No transecto $\mathrm{T} 10$, as dez principais espécies somam $50 \%$ dos indivíduos amostrados e Protium apiculatum (IVI=23,63), Parkia panurensis (IVI=21,5), Oenocarpus bataua (IVI=21,5), Macrolobium acaciefolium (IVI=7,7) e Qualea paraensis (IVI $=7,7$ ) somam $48 \%$ da dominância relativa (Tab. 10); essas duas últimas espécies apresentaram apenas um individuo, mas com grande área basal.

Nos transectos de mata alagada (T3 e T9) as dez principais espécies somam 76 e $87 \%$ do valor total do IVI, respectivamente (Tabs. 12, 13). No transecto T3, Pseudobombax cf. faroense, Qualea paraensis, Virola surinamensis e Clusia $c f$. planchoniana somam $46 \%$ do total do IVI; essas espécies representam $41 \%$ dos indivíduos amostrados e apenas as três primeiras representam $60 \%$ da

Tabela 12. Parâmetros fitossociológicos das espécies inventariadas no transecto T3 (Mata alagada).

\begin{tabular}{lrrrrr}
\hline Espécie & $\mathrm{n}$ & $\mathrm{DR}(\%)$ & $\mathrm{Fr}(\%)$ & $\mathrm{Dor}(\%)$ & $\mathrm{IVI}$ \\
\hline Pseudobombax cf. faroense & 36 & 18,0 & 14,65 & 22,66 & 55,31 \\
Qualea paraensis & 20 & 10,0 & 8,92 & 16,40 & 35,32 \\
Virola sp. & 8 & 4,0 & 4,46 & 21,40 & 29,86 \\
Clusia cf. planchoniana & 18 & 9,0 & 8,28 & 1,89 & 19,17 \\
Protium heptaphyllum & 15 & 7,5 & 8,28 & 3,34 & 19,12 \\
Qualea acuminata & 11 & 5,5 & 6,37 & 3,07 & 14,93 \\
Graffenrieta floribunda & 13 & 6,5 & 7,01 & 1,36 & 14,87 \\
Ferdinandusa guianensis & 12 & 6,0 & 6,37 & 1,51 & 13,88 \\
Luehea speciosa & 11 & 5,5 & 5,10 & 2,72 & 13,31 \\
Ilex inundata & 10 & 5,0 & 5,10 & 1,07 & 11,16 \\
Oxandra xylopioides & 7 & 3,5 & 3,18 & 2,64 & 9,32 \\
Mauritia flexuosa & 1 & 0,5 & 0,64 & 7,12 & 8,25 \\
Pagamea coriacea & 4 & 2,0 & 2,55 & 1,25 & 5,80 \\
Brosimum potabile & 2 & 1,0 & 1,27 & 2,86 & 5,13 \\
Sloanea grandiflora & 4 & 2,0 & 1,91 & 1,17 & 5,08 \\
Caryodendron amazonicum & 3 & 1,5 & 1,91 & 1,62 & 5,03 \\
Dulacia guianensis & 5 & 2,5 & 1,91 & 0,60 & 5,01 \\
Symphonia globulifera & 3 & 1,5 & 1,91 & 0,37 & 3,78 \\
Retiniphyllum truncatum & 3 & 1,5 & 1,91 & 0,30 & 3,71 \\
Clusia pana-panari & 1 & 0,5 & 0,64 & 2,42 & 3,56 \\
Myrciaria dubia & 2 & 1,0 & 1,27 & 0,41 & 2,68 \\
Nectandra lucida & 1 & 0,5 & 0,64 & 1,41 & 2,54 \\
Elaeoluma glabrascens & 2 & 1,0 & 1,27 & 0,18 & 2,45 \\
Brosimum acutifolium & 1 & 0,5 & 0,64 & 1,24 & 2,37 \\
Hebepetalum humirifolium & 2 & 1,0 & 0,64 & 0,40 & 2,04 \\
Neea ovalifolia & 1 & 0,5 & 0,64 & 0,14 & 1,27 \\
Macairea pachyphylla & 1 & 0,5 & 0,64 & 0,14 & 1,27 \\
Pseudomedia laevigata & 1 & 0,5 & 0,64 & 0,14 & 1,27 \\
Tabebuia insignis & 1 & 0,5 & 0,64 & 0,10 & 1,24 \\
Inga marginata & 1 & 0,5 & 0,64 & 0,09 & 1,22 \\
Total & 200 & 100 & 100 & 100 & 300 \\
\hline & & & & & \\
\hline
\end{tabular}


Tabela 13. Parâmetros fitossociológicos das espécies inventariadas no transecto T9 (Mata alagada).

\begin{tabular}{lrrrrr}
\hline Espécie & $\mathrm{n}$ & $\mathrm{Dr}(\%)$ & $\mathrm{Fr}(\%)$ & $\operatorname{Dor}(\%)$ & IVI \\
\hline Macrolobium angustifolium & 60 & 30,0 & 25,50 & 43,39 & 98,89 \\
Ferdinandusa guiaensis & 50 & 25,0 & 20,81 & 18,83 & 64,64 \\
Clusia cf. planchoniana & 21 & 10,5 & 9,40 & 4,96 & 24,86 \\
Pseudobombax cf. faroense & 10 & 5,0 & 6,71 & 11,98 & 23,69 \\
Hebepetalum humirifolium & 12 & 6,0 & 7,38 & 2,12 & 15,50 \\
Matayba inelegans & 4 & 2,0 & 2,68 & 5,54 & 10,22 \\
Protium heptaphyllum & 5 & 2,5 & 2,68 & 1,27 & 6,46 \\
Virola sp. & 3 & 1,5 & 2,01 & 2,71 & 6,23 \\
Rapanea guianensis & 4 & 2,0 & 2,68 & 1,28 & 5,97 \\
Licania gardneri & 4 & 2,0 & 2,68 & 1,22 & 5,91 \\
Nectandra lucida & 3 & 1,5 & 2,01 & 2,23 & 5,74 \\
Schefflera psilophylla & 4 & 2,0 & 2,68 & 0,53 & 5,22 \\
Qualea acuminata & 3 & 1,5 & 2,01 & 1,31 & 4,82 \\
Macairea pachyphylla & 3 & 1,5 & 2,01 & 0,29 & 3,80 \\
Hedyosmum brasiliense & 3 & 1,5 & 2,01 & 0,27 & 3,79 \\
Lueheopsis cf. rosea & 3 & 1,5 & 1,34 & 0,76 & 3,61 \\
Retiniphyllum truncatum & 2 & 1,0 & 1,34 & 0,33 & 2,67 \\
Sloanea grandiflora & 1 & 0,5 & 0,67 & 0,26 & 1,43 \\
Pseudomedia laevigata & 1 & 0,5 & 0,67 & 0,20 & 1,37 \\
Oenocarpus sp. & 1 & 0,5 & 0,67 & 0,15 & 1,32 \\
Cybianthus amplus & 1 & 0,5 & 0,67 & 0,13 & 1,30 \\
Qualea wittrockii & 1 & 0,5 & 0,67 & 0,11 & 1,28 \\
Chanouchiton kapleri & 1 & 0,5 & 0,67 & 0,11 & 1,28 \\
Total & 200 & 100 & 100 & 100 & 300 \\
\hline
\end{tabular}

dominância relativa (Tab. 12). No transecto T9, apenas Macrolobium angustifolium e Ferdinandusa guianensis somam $54 \%$ do total do IVI; essas espécies representam $55 \%$ dos individuos amostrados e $62 \%$ da dominância relativa (Tab. 12).

\section{Similaridade Florística e Estrutural}

A maioria (71\%) dos pares de transectos analisados apresentaram baixa similaridade florística $(\mathrm{J}<0,3)$. A similaridade entre os transectos no mesmo tipo fisionômico foram entre 0,3 e 0,4 . A fisionomia de mata alagada apresentou as menores similaridades com os outros tipos fisionômicos $(\mathrm{J}<0,07)$, exeção ao transecto T10 (mata ciliar) que apresentou indices de similaridade maiores com os transectos de floresta de terra firme (Tab. 14).

A análise de agrupamento, utilizando dados de abundância das espécies também confirma os índices de Jaccard, separando as três fisionomias (Fig. 2) e incluindo o transecto T10 no grupo de floresta de terra firme. O transecto $\mathrm{T} 10$ pode representar um gradiente entre mata ciliar e floresta de terra firme ou é um ponto onde a floresta de terra firme chega até a margem do Rio com fisionomia semelhante à mata ciliar. 
Tabela 14. Matriz de similaridade entre os transectos, usando índice de Jaccard em matriz binária de 207 espécies. $\mathrm{FTF}=$ floresta de terra firme; $\mathrm{MC}=$ mata ciliar; $\mathrm{MA}=$ mata alagada.

\begin{tabular}{|c|c|c|c|c|c|c|c|c|c|c|}
\hline & T1-FTF & T5-FTF & T8-FTF & T2-MC & T4-MC & T6-MC & $T 7-M C$ & T10-MC & T3-MA & T9-MA \\
\hline $\mathrm{T} 1 \mathrm{FTF}$ & . & & & & & & & & & \\
\hline T5-FTF & 0,396 & - & & & & & & & & \\
\hline T8-FTF & 0,350 & 0,356 & - & & & & & & & \\
\hline T2-MC & 0,208 & 0,253 & 0,179 & - & & & & & & \\
\hline T4-MC & 0,250 & 0,341 & 0,218 & 0,325 & . & & & & & \\
\hline T6-MC & 0,186 & 0,194 & 0,204 & 0,291 & 0,329 & . & & & & \\
\hline T7-MC & 0,255 & 0,263 & 0,247 & 0,350 & 0,377 & 0,427 & - & & & \\
\hline T10-MC & 0,372 & 0,379 & 0,389 & 0,253 & 0,330 & 0,255 & 0,292 & . & & \\
\hline T3-MA & 0,067 & 0,054 & 0,042 & 0,036 & 0,063 & 0,012 & 0,051 & 0.069 & - & \\
\hline T9-MA & 0,035 & 0,022 & 0.034 & 0,000 & 0,027 & 0,000 & 0,042 & 0,024 & 0,293 & . \\
\hline
\end{tabular}

\section{I S T Â N Cl A}

FTF-T1

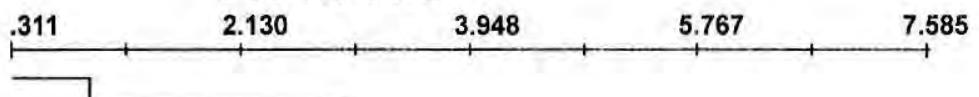

HC-T10

FTF-T5

FTF-T8

HC-T2

HC-T7

HC-T6

HC-T4

HA-T3

HA-T9

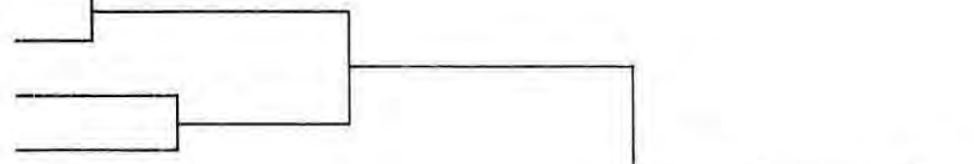

Figura 2. Dendrograma de dissimilaridade de dez transectos estudados em três fisionomias no Rio Comemoração, utilizando a abundância de 207 espécies, o método de Wards e a distância Euclidiana. 
A grande maioria (92\%) das espécies amostradas foram encontradas em apenas um tipo fisionômico; apenas 30 espécies estavam em dois ou mais ambientes. Segundo Wilson et al. (1996b), condições ambientais homogêneas proporcionam a sobrevivência de grupos similares de espécies e condições heterogêneas determinam grupos diferentes de espécies. Esses resultados revelam a grande heterogeneidade florística e estrutural existente dentro e entre os diferentes tipos físionômicos estudados e estão de acordo com vários estudos que mostram a heterogeneidade florística encontrada na Amazônia (Amaral, 1997; Campbell et al., 1986; Tello, 1995).

\section{CONCLUSÕES}

Na região do Rio Comemoração três fisionomias dominam: floresta de terra firme, com dossel alto e rica em espécies; mata ciliar, com dossel médio e troncos maiores; e, mata aberta alagada, com dossel baixo e pobre em espécies vegetais. A diversidade, em geral, é menor do que em outras florestas amazônicas, mas comparável a outras áreas de tensão ecológica na transição floresta/savana.

Nas florestas de terra firme e mata ciliar, espécies comumente encontradas nas florestas amazônicas foram as mais importantes, tais como: Qualea paraensis, Protium apiculatum, Tachigalia paniculata, Macrolobium acaciefolium, Dialium guianense, Protium heptaphyllum e
Swartzia arborescens nas florestas de terra firme e Maquira guianensis, Nectandra amazonium, Parkia panurensis, Zygia latifolia nas matas ciliares. Nas áreas de mata alagada, sujeitas a maior estresse hidrico, poucas espécies dominam, entre elas, Pseudobombax cf. faroense, Virola surinamensis, Clusia cf. planchoniana, Macrolobium angustifolium e Ferdinandusa guianensis.

A baixa similaridade florística entre o conjunto de transectos estudados reforça a existência de tipos fisionômicos distintos, com a similaridade baixa dentro das fisionomias sugerindo uma grande heterogeneidade florística.

\section{AGRADECIMENTOS}

A pesquisa recebeu apoio do herbário do Museu Paraense Emílio Goeldi, onde as amostras foram depositadas. Agradeço a colaboração e disposição do Carlos Roberto de Souza (mateiro), Nelson Rosa (identificador), Mauro (prensador), Evaldo (trepador), Paulo e Gerson (picadeiros), Sr. Gama (barqueiro), sem eles esse trabalho seria impossível de se realizar. Agradeço aos funcionários da Apidiá Planejamento Agropecuário Ltda, especialmente a Edgar M. Cardoso, Edmundo Machado Neto e Alcione Swinka Ferreira, que procuraram facilitar todas as etapas desse trabalho.

\section{Bibliografia citada}

Amaral, I,L. 1996. Diversidade floristica em floresta de terra firme na região do Rio 
Urucu-AM. Dissertação de mestrado, Instituto Nacional de Pesquisas da Amazônia/ Fundação Universidade do Amazonas, Manaus. 104p.

Brower, J.E.; Zar, J.H.; van Ende, C.N. 1998. Field and Laboratory Methods for General Ecology, 4a. ed. WCB/McGraw, New York. 273p.

Bulla, L. 1994. An index of evenness and its associated diversity measure. Oikos, 70:167-171.

Campbell, D.G.; Daly, D.C.; Prance, G.T.; Maciel, U.N. 1986, Quantitative ecological inventory of terra firme and varzea tropical forest on the Rio Xingu, Brazilian Amazon. Brittonia, 38(4):369-393.

Cottam, G.; Curtis, J.T. 1956. The use of distance measure in phytosociological sampling. Ecology, 37:451-460.

Coutinho, L.M. 1978. O Conceito do Cerrado. Revta. Brasil. Bot, 1:17-23.

Hartshorn, G.S. 1978. Tree falls and Tropical Forest Dynamics. In: Tomlinson, P.B.; Zimmermann, M.H. (eds.) Tropical Tree as Living Systems. Cambridge University Press, Cambridge, pp. 617-638.

IBGE, 1993. Mapa de Vegetação do Brasil. IBGE/IBAMA, Rio de Janeiro.

Keel, S.H. \& Prance, G.T. 1979. Studies of the vegetation of a white-sand black-water igapó (Rio Negro, Brasil), Acta Amazonica, 9:645-655.

Ludwig, J.A. \& Reynolds, J.F. 1988. Statisti-. cal Ecology: a primer on methods and computing. John Wiley \& Sons, New York. 337p.

Magurran, A.E. 1988. Ecological Diversity and its Measurement. CROOM HELM, London. 179p.

Milligan, G.W. \& Cooper, M.C. 1987. Methodology Review: Clustering Methods. Applied Psychological Measurement, 2:329-354.

Morellato, C.L.P.; Rosa, N.A. 1991. Caracterização de alguns tipos de vegetação na região Amazônica, Serra dos
Carajás, Pará, Brasil, Revta. Brasil. Bot, 14:1-14.

Nimer, E. 1991. Clima. In: IBGE. Geografia do Brasil, Região Norte, Vol.3, IBGE, Rio de Janeiro. pp. 61-71.

Pielou, E.C. 1977. Mathematical Ecology. Wiley, New York. 385p.

Pollard, J.H. 1971. On distance estimation of density in randomly distributed forest. Biometrics, 27:991-1002.

Prance, G.T.; Rodrigues, W.A.; Silva, M.F. 1976. Inventário florestal de um hectare de mata de terra firme, $\mathrm{km} 30$ da Estrada Manaus-Itacoatiara, Acta Amazonica, 6(1):9-35.

RADAMBRASIL. $1978 . \quad$ Projeto RADAMBRASIL. Folha SC.20 Porto Velho. Vol.16. Departamento Nacional da Produção Mineral - DNPM. Rio de Janeiro.

Silva, E.L.S. 1993. Inventário Preliminar das espécies arbóreas das florestas dos arredores de Boa Vista (Roraima) - Uma abordagem Fitossociológica. Dissertação de Mestrado, Instituto Nacional de Pesquisas da Amazônia/ Fundação Universidade do Amazonas, Manaus. $194 \mathrm{p}$.

Tello, J.R. 1995. Aspectos fitossociológicos das comunidades vegetais de uma toposeqüencia da Reserva Florestal Ducke do INPA. Tese de Doutorado, Instituto Nacional de Pesquisas da Amazônia/ Fundação Universidade do Amazonas, Manaus. 335p.

Veloso, H. P., Rangel Filho, A.L.R. \& Lima, J.C.A. 1991. Classificação da Vegetação Brasileira, adaptada a um sistema universal. IBGE, Rio de Janeiro. 123p.

Whitmore, T.C. 1975. Tropical Rain Forest of Far East. Claredon Press, Oxford. 282p.

Wilson, J.B, Wells, T.C., Trueman, I.C., Jones, G., Atkinson, M.D., Crawley, M.J., Dood, M.E.; Silvertown, J. 1996a. Are there assembly rules for plant species abundance? An investigation in relation to soil resources and successional trends. Journal 
of Ecology, 84:527-538.

Wilson, J.B., Ullmann, I.; Bannister, P. 1996b.

Do species assemblages ever recur? Ecology, 84:471-474. 